Supporting information

\title{
A Distinct Spin Structure and Giant Baromagnetic Effect in MnNiGe
}

\section{Compounds with Fe-Doping}

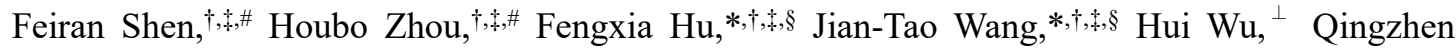
Huang, ${ }^{\perp}$ Jiazheng Hao, ${ }^{\dagger, \|}$ Zibing Yu, ${ }^{\dagger,+}$ Yihong Gao, ${ }^{\dagger,+}$ Yuan Lin, ${ }^{\dagger,+}$ Yangxin Wang, ${ }^{\dagger, \|}$ Cheng Zhang, ${ }^{\dagger, \star}$ Zhuo Yin, ${ }^{\dagger, *}$ Jing Wang, ${ }^{* \dagger,+, \nabla}$ Sihao Deng,,$\|$ Jie Chen, Lunhua He, ${ }^{*},,,, \|$ Tianjiao Liang, $\|$ Ji-

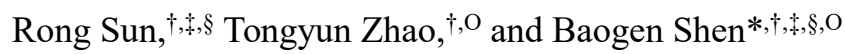

†nstitute of Physics, Chinese Academy of Sciences, Beijing 100190, China

tSchool of Physical Sciences, University of Chinese Academy of Sciences, Beijing 101408, China ${ }^{\S}$ Songshan Lake Materials Laboratory, Dongguan, Guangdong 523808, China

${ }^{\perp}$ NIST Center for Neutron Research, National Institute of Standards and Technology, Gaithersburg, Maryland 20899, USA

"Spallation Neutron Source Science Center, Dongguan 523803, China

${ }^{\nabla}$ Fujian Innovation Academy, Chinese Academy of Sciences, Fuzhou, Fujian 350108, China

${ }^{\mathrm{o}}$ Ganjiang Innovation Academy, Chinese Academy of Sciences, Ganzhou, Jiangxi, 341000, China

Series title:

SI-1. Unit conversion of baromagnetic effect for antiperovskite $\mathbf{M n}_{3} \mathbf{G a}_{0.95} \mathbf{N}_{0.94}$

SI-2. Neutron powder diffraction (NPD) studies

SI-3. Occupations of $\mathrm{Fe}$ atoms in $\mathrm{Mn}_{0.87} \mathrm{Fe}_{0.13} \mathrm{NiGe}$

SI-4. Computational methods of first-principles calculations

SI-5. Spherical coordinate to describe the spiral spin structure

SI-6. AFM spin configuration of stoichiometric MnNiGe

SI-7. Correlations of Mn-Mn distances with spin structure

SI-8 NPD measurements under variable magnetic fields

SI-9 Calculation for $\Delta M_{\mathrm{HP}}$ (BME) and baromagnetic coefficient $d$

SI-10 NPD measurements under variable pressure 


\section{SI-1. Unit conversion of baromagnetic effect for antiperovskite $\mathbf{M n}_{3} \mathbf{G a}_{0.95} \mathbf{N}_{\mathbf{0 . 9 4}}$}

Previously, Shi et al reported a large baromagnetic effect (BME) in $\mathrm{Mn}_{3} \mathrm{Ga}_{0.95} \mathrm{~N}_{0.94}{ }^{[\mathrm{S} 1]}$ The largest change of macroscopic magnetism was observed at $130 \mathrm{~K}$, from $0 \mu_{\mathrm{B}} /$ f.u. at $\Gamma^{5 \mathrm{~g}} \mathrm{AFM}$ phase to 0.63 $\mu_{\mathrm{B}} /$ f.u. at M-1 FIM phase under $7.5 \mathrm{kbar}$ pressure. The BME can be described by the difference between the magnetic moments at different pressures, i.e. $\Delta \mathrm{M}_{\mathrm{HP}}$,

$$
\Delta \mathrm{M}_{\mathrm{HP}}=\mathrm{M}(\mathrm{H}, \mathrm{P})_{T}-\mathrm{M}\left(\mathrm{H}, \mathrm{P}_{0}\right)_{T}
$$

where $\mathrm{P}_{0}$ stands for the atmospheric pressure (0kbar labeled thereafter) and $\mathrm{M}(\mathrm{H}, \mathrm{P})_{\mathrm{T}}$ is the magnetization under magnetic field $(\mathrm{H})$ and pressure $(\mathrm{P})$ at selected temperature $(\mathrm{T})$. Therefore, the maximum BME for the $\mathrm{Mn}_{3} \mathrm{Ga}_{0.95} \mathrm{~N}_{0.94}$ is $0.63 \mu_{\mathrm{B}} /$ f.u. at $130 \mathrm{~K}$ under $7.5 \mathrm{kbar}$. Based on unit conversion relation, i.e. $1 \mu_{\mathrm{B}} \approx 9.27 \times 10^{-21} \mathrm{emu}$, the $0.63 \mu_{\mathrm{B}} / \mathrm{f}$.u. can be converted into $14.4 \mathrm{emu} / \mathrm{g}$.

To quantify the sensitivity of BME as a sensor, the corresponding baromagnetic coefficient (BMC) $d^{[\mathrm{S} 2]}$ is further calculated as follows,

$$
\mathrm{d}=\left|\Delta \mathrm{M}_{\mathrm{HP}} / \Delta \mathrm{P}\right|_{\mathrm{H}, \mathrm{T}}
$$

where $\Delta \mathrm{P}$ stands for the applied pressure, i.e. $7.5 \mathrm{kbar}$ here. Then, the corresponding BMC is $d \sim 1.92 \mathrm{emu} \cdot \mathrm{g}^{-1} \cdot \mathrm{kbar}^{-1}$.

\section{SI-2. Neutron powder diffraction (NPD) studies}

The employed $\mathrm{Mn}_{0.87} \mathrm{Fe}_{0.13} \mathrm{NiGe}$ alloy was fabricated by conventional arc melting technique followed by annealing at $875^{\circ} \mathrm{C}$ for 6 days, and then quenching in liquid nitrogen. ${ }^{[\mathrm{S} 3]}$ To compare with the NPD results, macroscopic magnetic measurements under hydrostatic pressure were performed using a superconducting quantum interference device magnetometer (MPMS-7T) in a $\mathrm{BeCu}$ cylindrical pressure cell.

Neutron powder diffractions (NPD) under variable hydrostatic pressure, magnetic field, and temperature were performed at the NIST Center for Neutron Research on the BT-1 high-resolution neutron powder diffractometer. Ge (311) monochromator was used to produce a monochromatic neutron beam of wavelength $2.0775 \AA$. The cryostat device is the low T high power closed cycle refrigerators (CCR) with Sumitomo cold heads. The magnetic field generating device is the 7T vertical field magnet system with a top loading CCR insert. The pressure generating device is the HW-02 type aluminum pressure cell, and the pressing medium is helium gas.

The crystal and magnetic structures were refined by the Rietveld method using the General Structure Analysis System (GSAS) suite and the FullProf suite of programs. For the GSAS, we choose the cosine Fourier series with a leading constant term as the background fitting function, and employs a multi-term Simpson's rule integration as the peak profile fitting function. For the FullProf, we choose linear interpolation as the background fitting function, and employs the Thompson-CoxHasting pseudo-Voigt convoluted with axial divergence asymmetry function to fit the peak profile. As representatives, Fig.S1a, b show the NPD patterns and the refinements at $5 \mathrm{~K}$ and $200 \mathrm{~K}$, respectively, collected under atmospheric pressure. Fig.S1c displays the refined lattice parameters of orthorhombic structure with temperature at atmospheric pressure.

Figure $1 \mathrm{f}$ shows variable temperature NPD patterns of $\mathrm{Mn}_{0.87} \mathrm{Fe}_{0.13} \mathrm{NiGe}$ in the absence of pressure and magnetic field, where the incommensurate magnetic satellites (red), orthorhombic crystal reflections (green) and hexagonal crystal reflections (purple) are indexed. In the low temperature zone, a series of orthorhombic structure reflections and magnetic satellites can be observed. With increasing temperature, the peak intensity of $(000)^{ \pm}$magnetic satellite (red line in Fig.1e) shows a sharp increase around $T_{m} \sim 150 \mathrm{~K}$ (purple zone in Fig.1e), but the peak intensity of 
orthorhombic (101) crystal reflections (yellow line in Fig.1e) remains no anomaly until around $\sim 250 \mathrm{~K}$. This suggests that a transition of spin-structure occurs around the $T_{m}$ in the orthorhombic martensite region (purple zone in Fig.1d,e around 150K). NPD refinements determined the spinstructure to be CyS-AFM ${ }^{\mathrm{b}}$ and $70^{\circ}$-CoS-FM ${ }^{\mathrm{a}}$ below and above $T_{m}$, respectively.

With further increasing temperature up to $T_{m s t r u} \sim 250 \mathrm{~K}$, both the $(000)^{ \pm}$magnetic peak and the orthorhombic (101) crystal reflection drop sharply, while the hexagonal (002) crystal reflection appears (Fig.1f, green line in Fig.1e), indicating a concurrent magnetic and structural transition around the $T_{m s t r u}$ from the orthorhombic structure with incommensurate magnetic ordering to paramagnetic hexagonal structure (green zone in Fig.1e).

Figure 1d shows the refined Mn-Mn distances from Fig.1f in the orthorhombic martensitic region. One can notice the step change of $\mathrm{Mn}-\mathrm{Mn}(\mathrm{d} 1)$ and $\mathrm{Mn}-\mathrm{Mn}(\mathrm{d} 2)$ distances around the $T_{m}$. The pink and yellow zones below and above $T_{m}$ correspond to CyS-AFM ${ }^{\mathrm{b}}$ (Fig.1a) and $70^{\circ}$-CoS-FM ${ }^{\mathrm{a}}$ (Fig.1b), respectively. 
a)

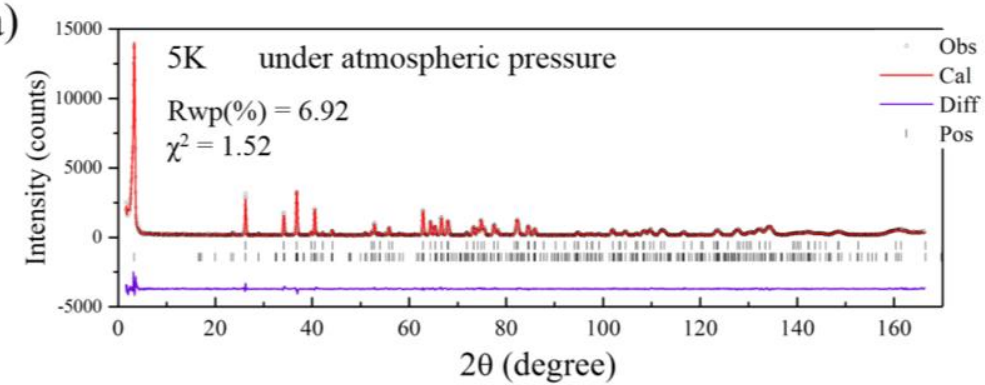

b)

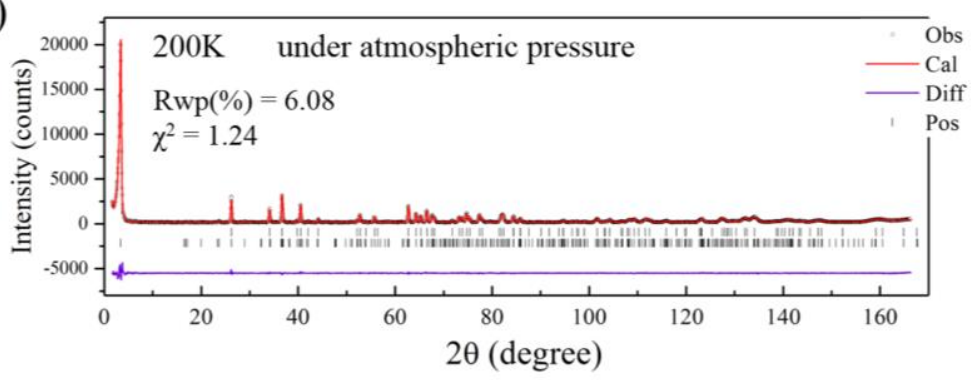

c)

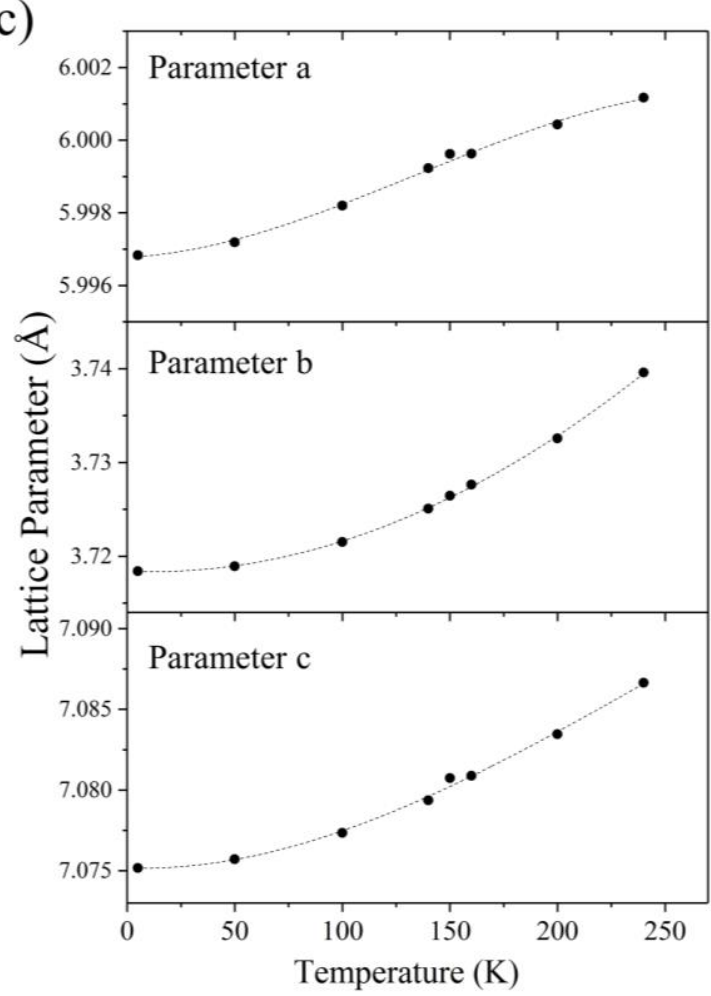

Fig.S1 Observed (black circle), calculated (red line) NPD patterns, their difference (purple line), peak position (black bar) for $\mathrm{Mn}_{0.87} \mathrm{Fe}_{0.13} \mathrm{NiGe}$ collected at a) $5 \mathrm{~K}$ and b) $200 \mathrm{~K}$, respectively. c) Refined lattice parameters of orthorhombic structure with temperature at atmospheric pressure.

\section{SI-3. Occupations of $\mathrm{Fe}$ atoms in $\mathrm{Mn}_{0.87} \mathrm{Fe}_{0.13} \mathrm{NiGe}$}

To check the titled composition $\mathrm{Mn}_{0.87} \mathrm{Fe}_{0.13} \mathrm{NiGe}$ and the occupations of introduced $\mathrm{Fe}$ atoms, atomic occupancies were refined based on NPD data. No obviously preferential orientations or any reflections of impure crystal phase were found in our NPD patterns. In order to eliminate the influence from the reflections of magnetic phase, we chose the NPD data collected at $300 \mathrm{~K}$, which lies in the paramagnetic hexagonal phase region. 
In the stoichiometric MnNiGe alloy with $\mathrm{Ni}_{2}$ In-type hexagonal structure (space group: $\mathrm{P} 63 / \mathrm{mmc}$ ), the $\mathrm{Mn}, \mathrm{Ni}$ and Ge atoms occupy the $2 \mathrm{a}(0,0,0), 2 \mathrm{~d}(1 / 3,2 / 3,3 / 4)$, and $2 \mathrm{c}(1 / 3,2 / 3,1 / 4)$ crystallographic positions, respectively. In order to refine the atomic occupancies for the Fe-doped MnNiGe alloy, we need to locate the substituted crystallographic position of the Fe element at first.

Firstly, we built an initial structural model of stoichiometric MnNiGe and made refinements with the atomic occupancy parameters fixed, labeled as the $1^{\text {st }}$ refinement. The observed and calculated intensity cannot fit well, especially on the hexagonal (110) peak (Fig.S2a), which indicates that the structural model is incomplete. Then, we improved the refinement by relaxing the atomic occupancy parameters, labeled as the $2^{\text {nd }}$ refinement. The observed and calculated intensity fit well, as shown in Fig.S2b. The refined atomic occupancies of $\mathrm{Ni}(100.5(3) \%)$ and $\mathrm{Ge}(100.2(4) \%)$ remain around $100 \%$, while the refined occupancy of $\mathrm{Mn}$ is changed to $57.6(5) \%$ (see Table S1). These refinement results indicate that, the coherent scattering length of $\mathrm{Ni}$ and $\mathrm{Ge}$ exactly fit the reflection intensities from the $2 \mathrm{~d}$ sites and $2 \mathrm{c}$ sites, respectively. (i.e., the $2 \mathrm{~d}$ sites and $2 \mathrm{c}$ sites are occupied only by the $\mathrm{Ni}$ atoms and the Ge atoms, respectively.) As for the 2a sites, the structural model with 57.6(5)\% Mn element occupied is obviously not in line with the fact, which suggests the refinement result to be false. In this situation, there probably be other element at the 2a sites to optimize the coherent scattering length. It means only the 2a sites (Mn sites) can be substituted by the Fe element.

Based on the analysis, we further improved the refinement by introducing the Fe element into the 2a sites, and relax all four atomic occupancy parameters, labeled as the $3^{\text {rd }}$ refinement. The observed and calculated intensities fitted very well (Fig.S2c), and the refinement results show that the occupancies of $\mathrm{Mn}$ at 2a sites, Fe at 2a sites, Ni at 2d sites and $\mathrm{Ge}$ at $2 \mathrm{c}$ sites are 87.5(3)\%, 12.5(3)\%, $100.1(6) \%$ and $99.8(7) \%$, respectively (Table S1). The refined occupancies of each element are close to the nominal composition $\mathrm{Mn}_{0.87} \mathrm{Fe}_{0.13} \mathrm{NiGe}$. Owing to the unchanged relative-site occupations during the diffusionless and displacesive martensitic transition, ${ }^{[\mathrm{S} 4]}$ all atoms consistently occupy their respective sites before and after the martensitic transition. So the titled composition $\mathrm{Mn}_{0.87} \mathrm{Fe}_{0.13} \mathrm{NiGe}$ is used in the entire article.

Table S1 The atomic occupancies at $2 \mathrm{a}$ sites, $2 \mathrm{~d}$ sites, and $2 \mathrm{c}$ sites from the $1^{\text {st }}, 2^{\text {nd }}$ and $3^{\text {rd }}$ refinement results, respectively.

\begin{tabular}{c|c|c|c|c}
\hline & $2 \mathrm{a}(0,0,0)$ & $2 \mathrm{~d}(1 / 3,2 / 3,3 / 4)$ & $2 \mathrm{c}(1 / 3,2 / 3,1 / 4)$ & Rwp(\%), $\chi^{2}$ \\
\hline $1^{\text {st }}$ refinement & Mn 100\% (fix) & Ni 100\% (fix) & Ge 100\% (fix) & $9.70,2.845$ \\
\hline $2^{\text {nd }}$ refinement & Mn 57.6(5)\% & Ni $100.5(3) \%$ & Ge $100.2(4) \%$ & $7.92,1.895$ \\
\hline $3^{\text {rd }}$ refinement & $\begin{array}{c}\text { Mn 87.5(3)\% } \\
\text { Fe } 12.5(3) \%\end{array}$ & Ni $100.1(6) \%$ & Ge 99.8(7)\% & $7.91,1.895$ \\
\hline
\end{tabular}


a)

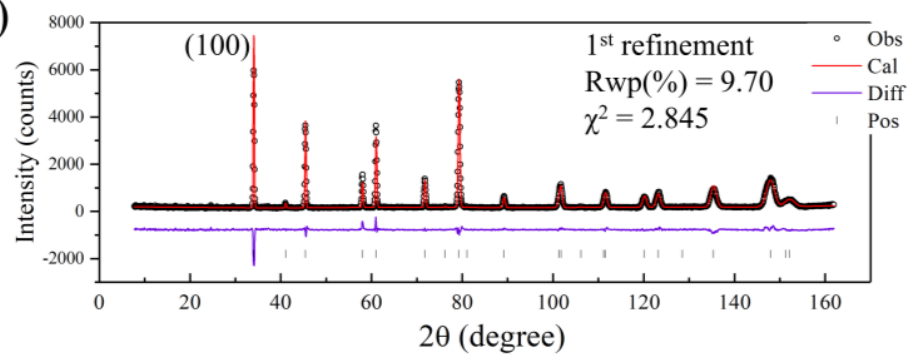

b)

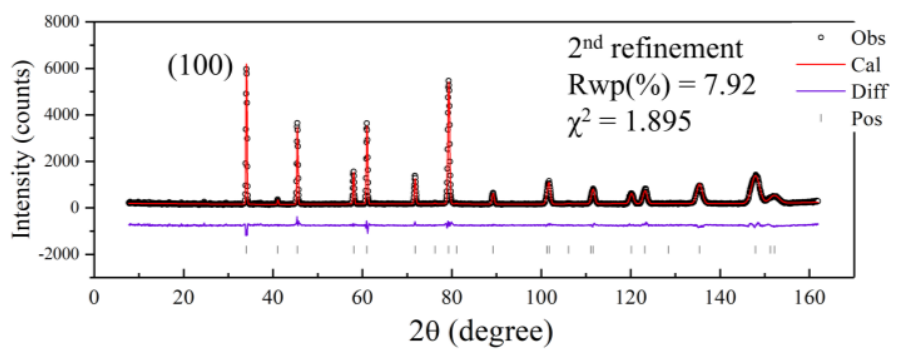

c)

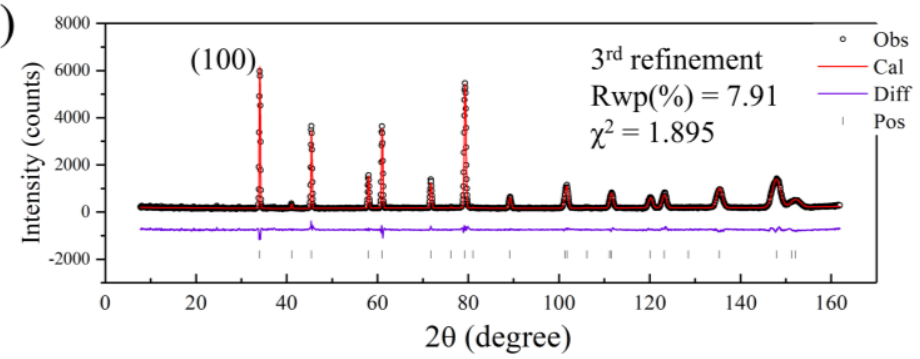

Fig.S2 Observed (black circle), calculated (red line) NPD patterns collected at 300K, their difference (purple line), peak position (black bar) of the a) $1^{\text {st }}$, b) $2^{\text {nd }}$ and c) $3^{\text {rd }}$ refinement results, for $\mathrm{Mn}_{0.87} \mathrm{Fe}_{0.13} \mathrm{NiGe}$.

\section{SI-4 Computational methods of first-principles calculations}

The density functional theory (DFT) calculations are performed using the Vienna ab initio simulation package $(\mathrm{VASP})^{[\mathrm{S5}, \mathrm{S6}]}$ with the projector augmented wave method ${ }^{[\mathrm{S7}, \mathrm{S} 8]}$ and spin polarized generalized gradient approximation ${ }^{[\mathrm{S} 9]}$ for the exchange-correlation energy. The valence states $3 d^{6} 4 s^{1}$ for $\mathrm{Mn}, 3 d^{7} 4 s^{1}$ for $\mathrm{Fe}, 3 d^{9} 4 s^{1}$ for $\mathrm{Ni}$, and $4 \mathrm{~s}^{2} 4 \mathrm{p}^{2}$ for Ge are used with the energy cutoff of $500 \mathrm{eV}$ for the plane wave basis set. We built the supercell of $\mathrm{Mn}_{0.87} \mathrm{Fe}_{0.13} \mathrm{NiGe}$ compound based on the atomic positions from NPD refinements, i.e., only Mn sites are occupied by the Fe atoms, and the occupation of $\mathrm{Fe}$ atoms is $12.5 \%$ (Table $\mathrm{S} 1$ ).

To simulate the energy of the different spiral magnetic structures in $\mathrm{Mn}_{0.87} \mathrm{Fe}_{0.13} \mathrm{NiGe}$ compound (Fig. 4a), a $6 \times 1 \times 1$ supercell (including $24 \mathrm{Mn}$ sites, $24 \mathrm{Ni}$ sites and $24 \mathrm{Ge}$ sites) is adopted, in which $3 \mathrm{Mn}$ sites are occupied by Fe atoms (equivalent $12.5 \% \mathrm{Mn}$ atoms substituted by Fe atoms, which is completely in line with the NPD refinements (Table S1)). The experimental lattice parameters from NPD refinements at $120 \mathrm{~K} / 6 \mathrm{kbar}(a=35.8862 \AA, b=3.7151 \AA$, and $c=7.0672 \AA)$ and the corresponding crystallographic positions $[\mathrm{Mn}(\mathrm{Fe})(0.0306,0.25,0.6798), \mathrm{Ni}(0.1461,0.25$, 0.0577), $\mathrm{Ge}(0.2591,0.25,0.3747)]$ were used for the calculations of CyS-AFM ${ }^{\mathrm{b}}$ and $45^{\circ}-\mathrm{CoS}_{-} \mathrm{FM}^{\mathrm{a}}$ spin structures (Fig.4a). The Brillouin zone is sampled with a $1 \times 12 \times 6$ Gamma-centered $k$-point grid. The energy convergence criterion is set at $10^{-6} \mathrm{eV}$ throughout the present calculations. The lattice compression and expansion are simulated with an isotropic lattice strain from $-1 \%$ to $+5 \%$ related to the experimental lattice parameters. 
To construct the spiral configuration of CyS-AFM ${ }^{\mathrm{b}}$ and $45^{\circ}$-CoS-FM ${ }^{\mathrm{a}}$, we need to convert the spherical coordinate of all spins in a complete magnetic cell into the coordinate of the space rectangular coordinate system at first. As the magnetic cell volume is about 6 times larger than the unit cell volume, a $6 \times 1 \times 1$ supercell (including $24 \mathrm{Mn}$ sites, $24 \mathrm{Ni}$ sites and $24 \mathrm{Ge}$ sites) is adopted, which means there are 24 spins in the magnetic cell.

For the CyS-AFM ${ }^{\mathrm{b}}$, all components of the magnetic moment lie in the a-c plane, so the magnetic moment component along the b-axis is 0 . Besides, the spins can be divided into 12 groups along aaxis as shown in Fig.S3. In each group, there are two parallel spins. From group 1 to group 12, the spins rotate about $30^{\circ}$ counterclockwise in the a-c plane each step, and return to the original direction at group 1' (the original direction of the next magnet cell period). Then, we can build a space rectangular coordinate system just based on the a-axis and c-axis (since the magnetic moment component along the b-axis is 0 , the b-axis can be ignored), and calculate the coordinates of these 12 spins, as shown in Fig.S4. Considering that the magnetic moment of the Mn site is about $2.8 \mu_{\mathrm{B}}$ at $120 \mathrm{~K}$, we set the modulus of the 12 spins to be $3 \mu_{\mathrm{B}}$.

For the $45^{\circ}-\mathrm{CoS}-\mathrm{FM}^{\mathrm{a}}$, we can divide the magnetic moment into two components, i.e. a spiral AFM component in b-c plane and a linear FM component along a-axis. For the former, the construction process is similar to the CyS-AFM ${ }^{\mathrm{b}}$ mentioned above. Then, though combining the $\mathrm{b}$ and c coordinates of the spiral AFM component with a coordinates of FM component, the $45^{\circ}$-CoS$\mathrm{FM}^{\mathrm{a}}$ can be constructed.

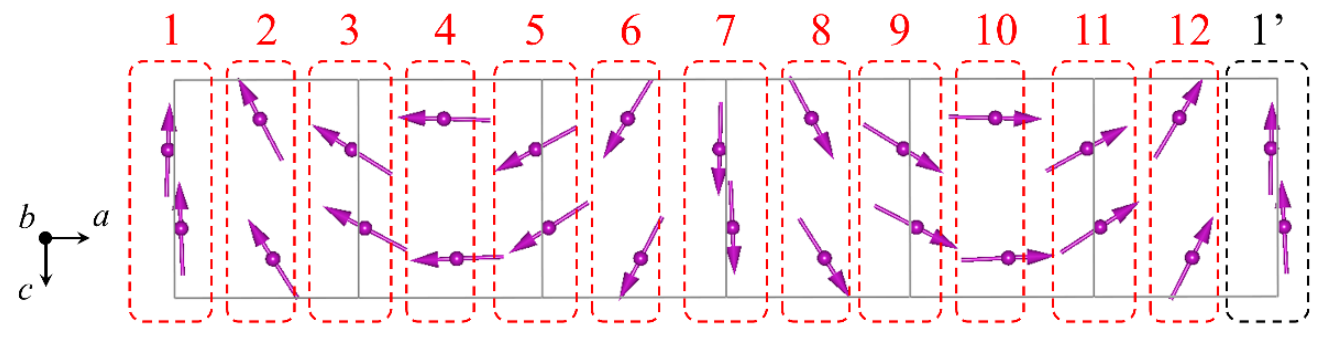

Fig.S3 The spiral spin structure of CyS-AFM ${ }^{\mathrm{b}}$ in the Fe-doped $\mathrm{Mn}_{0.87} \mathrm{Fe} 0.13 \mathrm{NiGe}$. 


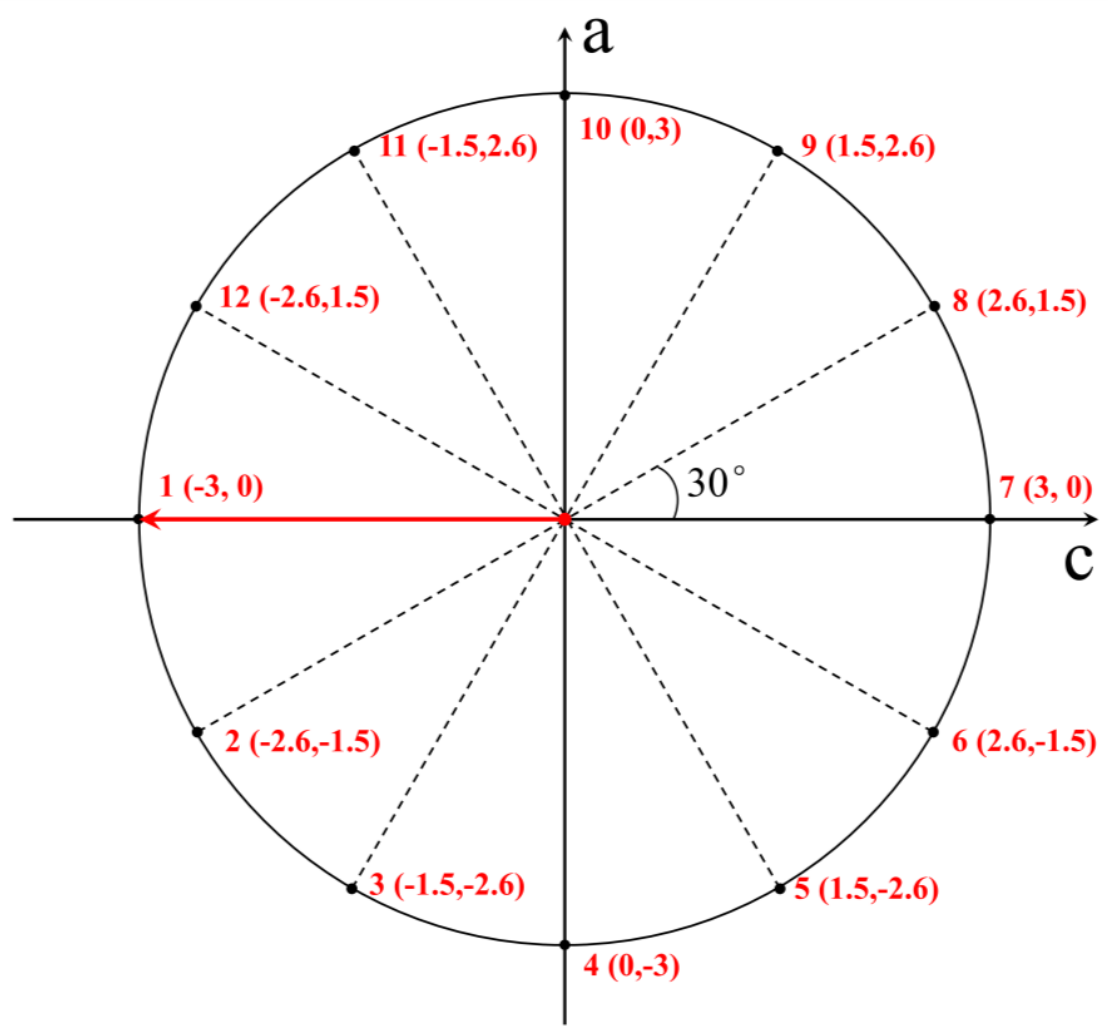

Fig.S4 The specific rectangular coordinates of each spin in CyS-AFM ${ }^{\mathrm{b}}$ of Fig.S3.

To simulate the electronic density of states (DOS) of the high and low spin states in $\mathrm{Mn}_{0.87} \mathrm{Fe}_{0.13} \mathrm{NiGe}$ compound (Fig. $4 \mathrm{~b}$ ), a $2 \times 1 \times 1$ supercell (including $8 \mathrm{Mn}$ sites, $8 \mathrm{Ni}$ sites and 8 Ge sites) is adopted, where $1 \mathrm{Mn}$ site are occupied by $\mathrm{Fe}$ atoms (equivalent $12.5 \% \mathrm{Mn}$ atoms substituted by Fe atoms, which is also completely in line with the NPD refinements (Table S1)). The experimental lattice parameters from NPD refinements at $5 \mathrm{~K} / 0 \mathrm{kbar}(a=11.9937 \AA, b=3.7184 \AA$, and $c=7.0751 \AA)$ and the corresponding crystallographic positions $[\mathrm{Mn}(\mathrm{Fe})(0.0344,0.25,0.6796)$, Ni $(0.1459,0.25,0.05817)$, Ge $(0.2583,0.25,0.3740)]$ were used for the calculation of high spin state $\left(\mathrm{M}_{\mathrm{Mn}(\mathrm{Fe})} \sim 3.06 \mu_{\mathrm{B}}\right)$ and low spin state $\left(\mathrm{M}_{\mathrm{Mn}(\mathrm{Fe})} \sim 2.37 \mu_{\mathrm{B}}\right)$. The Brillouin zone is sampled with a 4 $\times 12 \times 6$ Gamma-centered $k$-point grid. The energy convergence criterion is set at $10^{-6} \mathrm{eV}$ throughout the present calculations.

\section{SI-5. Spherical coordinate to describe spiral spin structure}

To accurately describe the evolution process of incommensurate spiral magnetic structure, spherical coordinate was used, as shown in Fig.S5 (Fig.1c). The spin vector $\boldsymbol{M}$ lies on the cone with a half-angle $\psi, \theta$ is the angle between the cone axis $l$ and the a axis, and $\varphi$ is the angle between the projection of $l$ on the b-c plane and the c axis. 


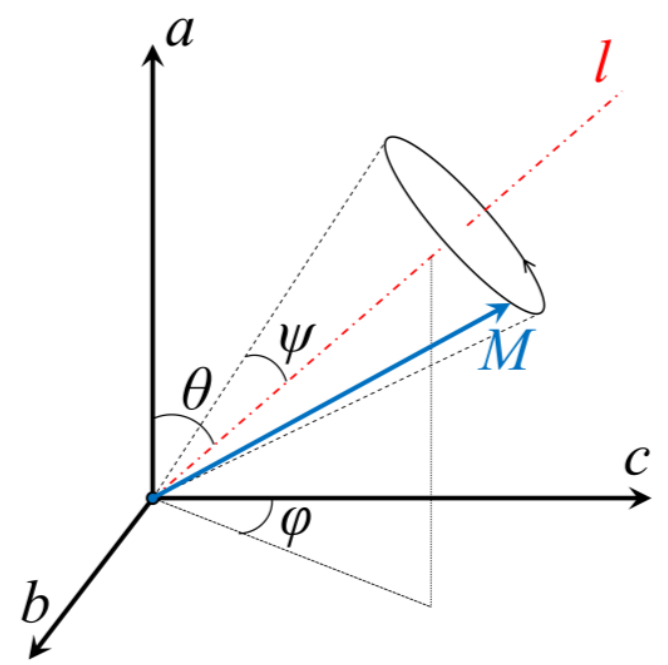

Fig.S5 Spherical coordinate to describe spiral spin structure

\section{SI-6. The AFM configuration of stoichiometric MnNiGe}

Bazela et $\mathrm{a}^{[\mathrm{S} 10]}$ investigated the magnetic structure of the stoichiometric $\mathrm{MnNiGe}$ via magnetic measurements and neutron diffraction. The stoichiometric MnNiGe undergoes a martensitic transition around $\mathrm{T}_{\mathrm{S}} \sim 420 \mathrm{~K}$, and the martensite displays spiral AFM structure with Neel temperature $\mathrm{T}_{\mathrm{N}}$ at $\sim 356 \mathrm{~K}$.

The neutron diffraction measurements were performed at 295K. The appearance of $(000)^{ \pm}$ magnetic satellite peaks, which can be indexed by the propagation vector $\mathrm{k}=[\tau, 0,0]$ with $\tau \sim 0.21$, indicating an incommensurate spiral magnetic structure. The refinement results showed that the magnetic moment localizes on $\mathrm{Mn}$ sites with $\mu(\mathrm{Mn})=2.2(1) \mu_{\mathrm{B}}$ at $295 \mathrm{~K}$, and the spiral axis lies along the a-axis, i.e. $\psi=90^{\circ}, \theta=0^{\circ}$, and $\varphi=0^{\circ}$, as shown in Fig. S4a, which was named as Simplespiral. ${ }^{[10]}$

Obviously, the formed cycloidal spiral with AFM coupling (abbreviated as CyS-AFM ${ }^{b}$ ) in the Fedoped $\mathrm{Mn}_{0.87} \mathrm{Fe}_{0.13} \mathrm{NiGe}$ in present work is different from the Simple-spiral spin structure. The optimal parameters are $\psi=90^{\circ}, \theta=90^{\circ}$, and $\varphi=90^{\circ}$, i.e. the cone axis $l$ is along the $\mathrm{b}$ axis, and the magnetic moment is perpendicular to the $\boldsymbol{l}$ for the CyS-AFM , as shown in Fig. S4b. Besides, the magnetic cell volume of $\mathrm{Mn}_{0.87} \mathrm{Fe}_{0.13} \mathrm{NiGe}$ is also different from that of stoichiometric MnNiGe. For the stoichiometric $\mathrm{MnNiGe}$, the $\tau$ is about 0.21 , which means the magnetic cell volume is about 5 times larger than the unit cell volume (lower panel of Fig.S6a). But for the $\mathrm{Mn}_{0.87} \mathrm{Fe}_{0.13} \mathrm{NiGe}$, the $\tau$ is about 0.167 , which means the magnetic cell volume is about 6 times larger than the unit cell volume (lower panel of Fig.S6b) 
a) Simple spiral

(AFM)

$\mathrm{MnNiGe}$

$$
\begin{aligned}
& \theta=0^{\circ} \\
& \varphi=0^{\circ} \\
& \psi=90^{\circ}
\end{aligned}
$$

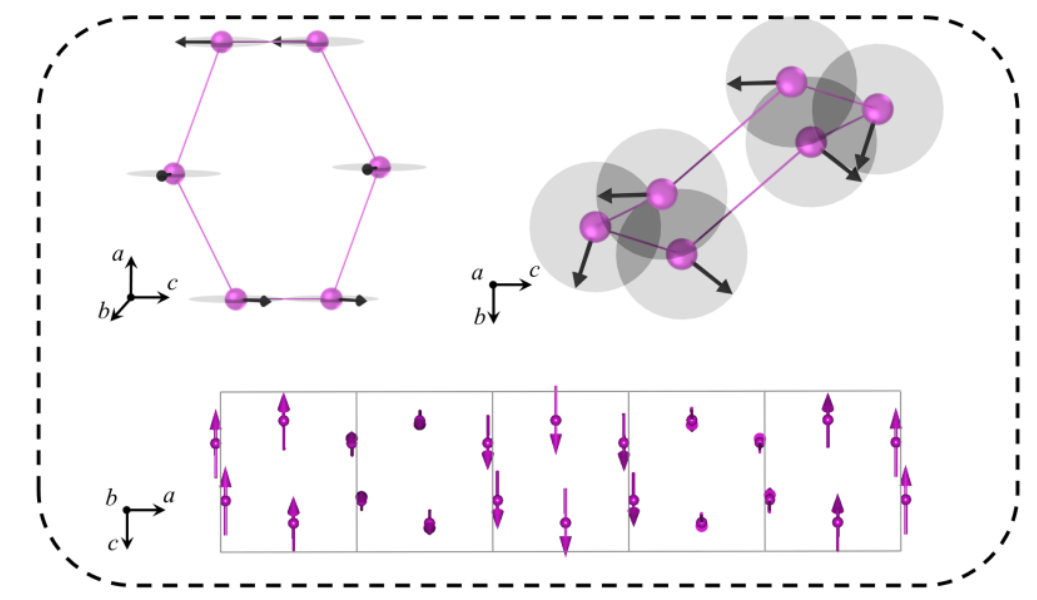

b) Cycloidal spiral $\left(\mathrm{CyS}-\mathrm{AFM}^{\mathrm{b}}\right)$ $\mathrm{Mn}_{0.87} \mathrm{Fe}_{0.13} \mathrm{NiGe}$

$\theta=90^{\circ}$
$\varphi=90^{\circ}$
$\psi=90^{\circ}$

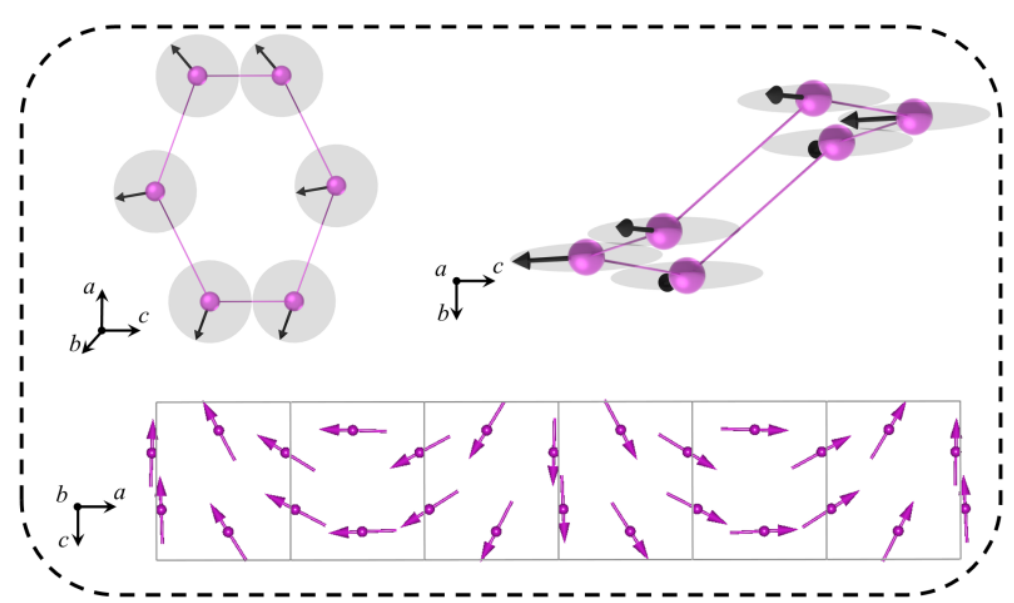

Fig. S6 The sketch of a) the Simple-spiral in the stoichiometric MnNiGe, and b) the formed cycloidal spiral spin structure with AFM coupling (abbreviated as CyS-AFM ${ }^{b}$ ) in the Fe-doped $\mathrm{Mn}_{0.87} \mathrm{Fe}_{0.13} \mathrm{NiGe}$.

\section{SI-7. Correlations of Mn-Mn distances with spin structure}

According to $J_{i j}=-\frac{2 m k_{F}{ }^{4}}{\pi h^{2}} J_{p d}^{2} F\left(2 k_{F} r_{i j}\right) \exp \left(-\frac{r_{i j}}{l}\right),{ }^{[111]}$ the change of the distance between the magnetic atoms $\left(r_{i j}\right)$ can change the spin structure via converting the exchange interaction $\left(J_{i j}\right)$. As studied earlier, for the Mn-based alloys with TiNiSi-type martensitic orthorhombic structure (space group: Pnma), the spin structure results from the competition between direct exchange of Mn-Mn(d1) and superexchange of Mn-Mn(d2) (Fig.1a), while the former plays a dominant role. ${ }^{[\mathrm{S} 12, \mathrm{~S} 13]}$

Regardless of the Mn-Mn(d2), variable temperature NPD refinements detected anomalous contraction of the Mn-Mn(d1) length (Fig.1d, Fig.S7) during the formation of new spin structure CyS-AFM ${ }^{\mathrm{b}}$ (AFM state) from the $70^{\circ}-\mathrm{CoS}_{-} \mathrm{FM}^{\mathrm{a}}$ (canted FM state) at around the transition $\mathrm{T}_{\mathrm{m}} \sim 150 \mathrm{~K}$, though no anomaly occurs in the lattice parameters (Fig.S1c). Furthermore, variable pressure NPD measurements were performed at $120 \mathrm{~K}$ in the $\mathrm{CyS}-\mathrm{AFM}^{\mathrm{b}}$ region. As the applied pressure is higher than $4 \mathrm{kbar}$, careful refinements detected a further contraction of Mn-Mn(d1) (Fig.3g, Fig.S7) during the spin configuration evolution from the new CyS-AFM ${ }^{\mathrm{b}}$ (AFM state) to the $45^{\circ}-\mathrm{CoS}_{-} \mathrm{FM}^{\mathrm{a}}$ (canted FM state), while no obvious anomaly appears in the lattice parameters (Fig.S9).

Previously, to study the effect of structural parameters on the magnetic ground state of the Mnbased orthorhombic (Pnma) alloys, Gercsi et al ${ }^{\left[{ }^{[12, S 13]}\right.}$ performed density functional theory (DFT) calculations on MnP binary alloy, which has the same orthorhombic (Pnma) structure as the Mn- 
based ternary alloys $\operatorname{MnMX}(\mathrm{M}=$ transition element, $\mathrm{X}=$ main element) by considering an additional atom occupied at a general $4 c$ crystallographic position. In $\mathrm{MnP}$, the $\mathrm{Mn}-\mathrm{Mn}(\mathrm{d} 1)$ with the direct exchange and the Mn-Mn(d2) with the superexchange through Mn-P-Mn were considered. Calculations indicated that the magnetic ground state of the $\mathrm{MnP}$ critically depends on the $\mathrm{Mn}$ $\mathrm{Mn}(\mathrm{d} 1)$ length. For $\mathrm{d} 1 \leq 2.66 \AA$, the close distance between $\mathrm{Mn}$ atoms leads to a strong overlap of $3 \mathrm{~d}$ orbitals, and no magnetic ground state is stable due to the broad $3 \mathrm{~d}$ hybrid bands. As the $\mathrm{d} 1$ length increases, the overlap of the $3 \mathrm{~d}$ orbitals of Mn becomes smaller, resulting in more localized $3 \mathrm{~d}$ electrons and enhanced exchanges between Mn atoms. For $2.66 \AA \leq \mathrm{d} 1 \leq 2.96 \AA$, the ground magnetic state favours FM coupling, i.e. the FM(1) region in Fig.S8, in line with the MnP (d1 $2.81 \AA$, FM state). With the d1 length increasing, the FM ground state is no longer stable, instead AFM coupling between Mn atoms is predicted for $2.96 \AA \leq \mathrm{d} 1 \leq 3.37 \AA$. With further increasing $\mathrm{d} 1$ length to $\mathrm{d} 1 \geq 3.37 \AA$, the FM state prevails again, i.e. the FM(1) region in Fig.S8. Furthermore, the calculated results were found also successfully work in the Mn-based ternary TiNiSi structure compositions, such as $\mathrm{MnNiSi}(\mathrm{d} 1 \sim 2.78 \AA \text {, FM state })^{[\mathrm{S} 14]}$ and $\mathrm{MnCoP}(\mathrm{d} 1 \sim 2.88 \AA \text {, FM state })^{[\mathrm{S} 15]}$ in the $\mathrm{FM}(1)$ region, $\mathrm{MnNiGe}(\mathrm{d} 1 \sim 3.2 \AA \text {, } \mathrm{AFM} \text { state })^{[\mathrm{S} 10]}$ in the $\mathrm{AFM}$ region, and $\mathrm{MnCoGe}(\mathrm{d} 1 \sim$ $3.4 \AA$, FM state $)^{[\mathrm{S} 16]}$ in the $\mathrm{FM}(2)$ region.

Interestingly, the above evolution tendency of magnetic ground state with varying d1 length predicted by DFT calculations also roughly works in our $\mathrm{Mn}_{0.87} \mathrm{Fe}_{0.13} \mathrm{NiGe}$ (Fig.S7), though the exact d1 length does not match each other (Fig.S7, Fig.S8). The Mn-Mn(d1) lengths of $\mathrm{Mn}_{0.87} \mathrm{Fe}_{0.13} \mathrm{NiGe}$ with variable temperature and pressure, as well as the corresponding spin configurations, are summarized in Fig.S7. One can note that the ground magnetic state of $\mathrm{Mn}_{0.87} \mathrm{Fe}_{0.13} \mathrm{NiGe}$ favours canted $\mathrm{FM}$ coupling $\left(45^{\circ}-\mathrm{CoS}-\mathrm{FM}^{\mathrm{a}}\right)$ at $\mathrm{d} 1 \leq 3.165 \AA$, while the spiral AFM coupling $\left(\mathrm{CyS}^{-} \mathrm{AFM}^{\mathrm{b}}\right)$ appears at larger d1, i.e. $3.165 \AA \leq \mathrm{d} 1 \leq 3.185 \AA$. With further increasing $\mathrm{d} 1$ length to $\mathrm{d} 1 \geq 3.185 \AA$, another canted $\mathrm{FM}$ state $\left(70^{\circ}-\mathrm{CoS}-\mathrm{FM}^{\mathrm{a}}\right)$ prevails. Such tendency is similar to the case in MnP (Fig.S8). Moreover, the d1 range of the spiral AFM state $(3.165 \AA \sim 3.185 \AA)$ in $\mathrm{Mn}_{0.87} \mathrm{Fe}_{0.13} \mathrm{NiGe}$ also lies in the AFM region in $\mathrm{MnP}(2.96 \AA \sim 3.37 \AA)$, but the range of former is much smaller. The cause might be relative to the introduced FM coupling between $\mathrm{Mn}$ and $\mathrm{Fe}$ atoms owing to the substitution of $\mathrm{Mn}$ by $\mathrm{Fe}$ in $\mathrm{Mn}_{0.87} \mathrm{Fe}_{0.13} \mathrm{NiGe}$, which destructs the intrinsic AFM order and affects the electronic structure and coupling. ${ }^{[\mathrm{I} 4]}$

In order to know the key factors that can lead to the substantial changes in magnetic ground state, Gercsi et al ${ }^{[S 13]}$ calculated the total density of electronic states (DOS, $N_{\text {Tot }}$ ) to investigate the main difference between the AFM state and the two FM states (FM1 and FM2) for MnCoP (FM1 state), MnCoGe (FM2 state), and $\mathrm{MnCoP}_{0.5} \mathrm{Ge}_{0.5}$ (AFM state). The results indicated that, the $N_{\text {Tot }}\left(E_{\mathrm{F}}\right)$ at Fermi level in the AFM state region is considerably larger than the FM1 and FM2 state regions, which makes ferromagnetism unstable and promotes an AFM arrangement as described by Barcza et al. ${ }^{[S 17]}$ Relatively, earlier study by Lizarraga et al ${ }^{[\mathrm{S} 18]}$ suggested that the formation of the hybridisation-derived pseudogap at the Fermi energy can also stabilize the AFM compared to FM state. 


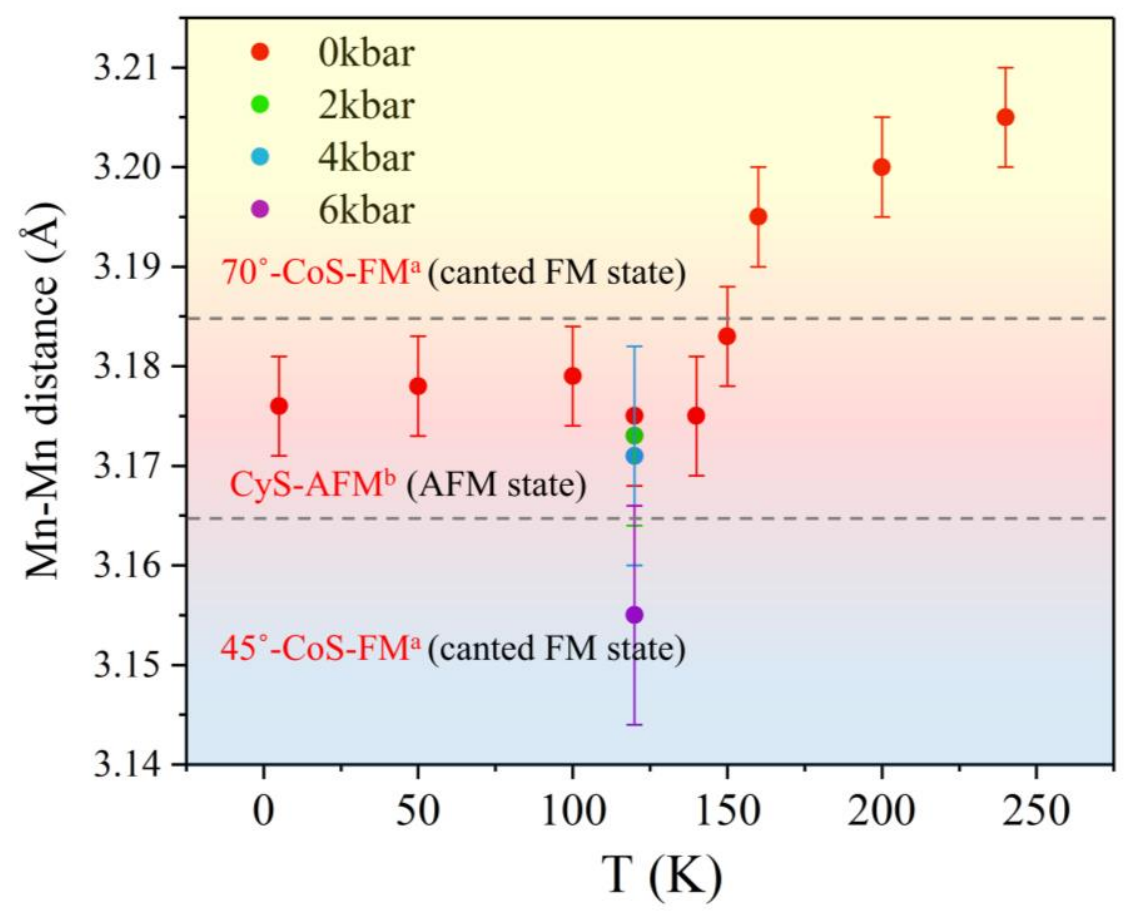

Fig. S7 Variation of the $\mathrm{Mn}-\mathrm{Mn}(\mathrm{d} 1)$ length with variable temperature and pressure, and the corresponding spin configurations, for $\mathrm{Mn}_{0.87} \mathrm{Fe}_{0.13} \mathrm{NiGe}$.

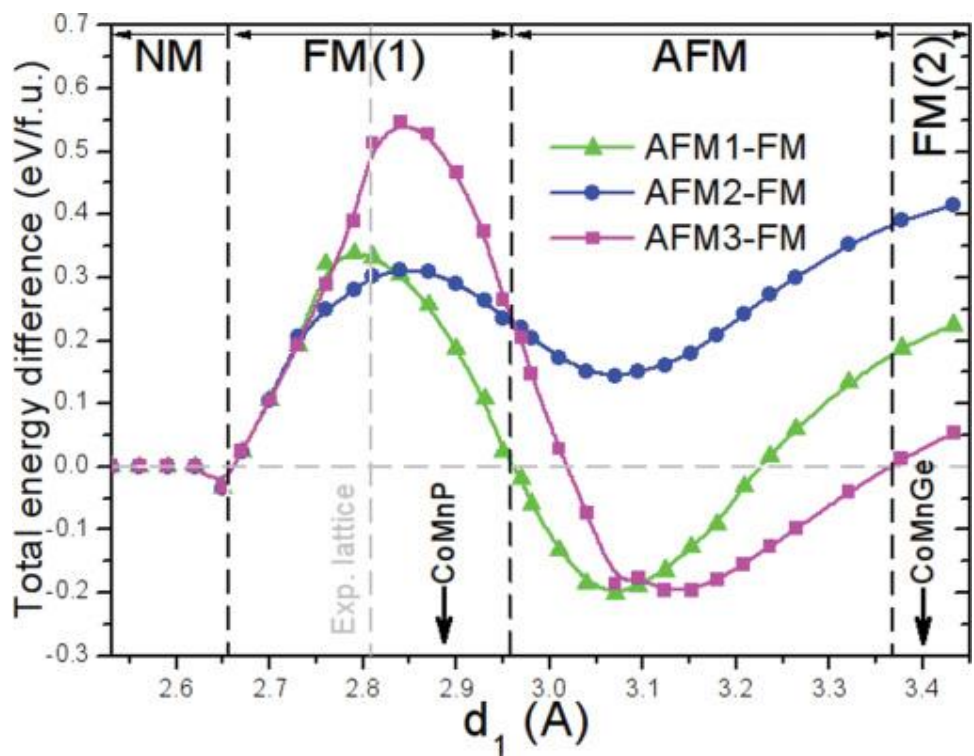

Fig. S8 DFT calculations for MnP with the same orthorhombic (Pnma) structure. DFT Stability of possible collinear magnetic structures, relative to ferromagnetism, within a single unit cell of $\mathrm{MnP}$ as a function of $d 1 \mathrm{Mn}-\mathrm{Mn}$ separation. AFM configurations become stable where $\Delta E_{\mathrm{AFM}-\mathrm{FM}}<0$. The vertical dashed line represents the experimental (strain free, $\varepsilon=0$ ) lattice of MnP. Reprinted figure 1 with permission from [Z. Gercsi, K. Hono, K. G. Sandeman, Physical Review B, 83, 174403, 2011]. Copyright (2011) by the American Physical Society. https://doi.org/10.1103/PhysRevB.83.174403. 


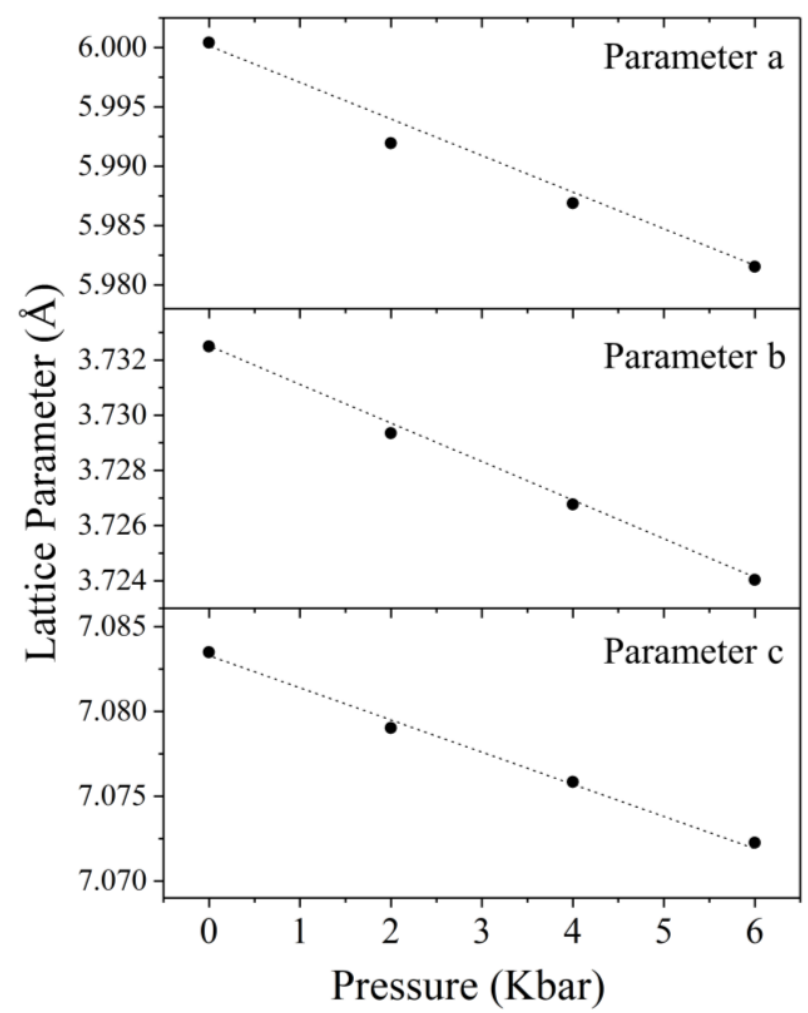

Fig.S9 Refined lattice parameters of orthorhombic structure with variable pressure at $120 \mathrm{~K}$ for $\mathrm{Mn}_{0.87} \mathrm{Fe}_{0.13} \mathrm{NiGe}$.

\section{SI-8 NPD measurements under variable magnetic fields}

Variable temperature NPD measurements indicate that, in the $150 \mathrm{~K}-250 \mathrm{~K}$ zone, the $\mathrm{Mn}_{0.87} \mathrm{Fe}_{0.13} \mathrm{NiGe}$ poses incommensurate conical spiral FM structure with $\psi=70^{\circ}, \theta=0^{\circ}$, and $\varphi=0^{\circ}$ (namely $70^{\circ}-\mathrm{CoS}-\mathrm{FM}^{\mathrm{a}}$, Fig. 1b). Below $150 \mathrm{~K}$, a new spiral spin-structure (Fig.1a) is developed, with $\psi=90^{\circ}, \theta=90^{\circ}$, and $\varphi=90^{\circ}$, i.e. the cone axis $\boldsymbol{l}$ is along the $\mathrm{b}$ axis, and the magnetic moment is perpendicular to the $\boldsymbol{l}$, corresponding to a cycloidal spiral structure with AFM nature (namely CyS$\left.\mathrm{AFM}^{\mathrm{b}}\right)$. While the magnetic moment is confined on $\mathrm{Mn}(\mathrm{Fe})$ sites with $\mu_{(\mathrm{Mn} / \mathrm{Fe})}=3.06(2) \mu_{\mathrm{B}}$ at $5 \mathrm{~K}$. Macroscopic magnetic measurements (M-H curves) at $5 \mathrm{~K}$ indicate that the CyS-AFM ${ }^{\mathrm{b}}$ behaves unstable against magnetic field, and a low critical magnetic field $\mathrm{H}_{\mathrm{C}} \sim 0.35 \mathrm{~T}$ can induce a metamagnetic transition with a step rise of magnetization(M), then $\mathrm{M}$ increases almost linearly until saturation at $4 \mathrm{~T}$ with saturated moment $\mathrm{M}_{\mathrm{S}} \sim 3.05 \mu_{\mathrm{B}}$.

To reveal the evolution process of the new CyS-AFM ${ }^{b}$ with magnetic field (H), NPD measurements under variable magnetic fields, especially around the $\mathrm{H}_{\mathrm{C}} \sim 0.35 \mathrm{~T}$, were performed at representative temperature 5K, as shown in Fig.2b(Fig.S10). The refined results are summarized in Table S2. As representatives, Fig.S11a and Fig.S11b display the refined 5K NPD patterns under $0.6 \mathrm{~T}$ and $5 \mathrm{~T}$, respectively. At $\mathrm{H}<0.6 \mathrm{~T}$, the sample remains the $\mathrm{CyS}-\mathrm{AFM}^{\mathrm{b}}$, but at around $\mathrm{H} \sim 0.6 \mathrm{~T}$, the CyS-AFM ${ }^{\mathrm{b}}$ evolves into another conical spiral FM with $\psi=70^{\circ}, \theta=90^{\circ}, \varphi=90^{\circ}\left(70^{\circ}\right.$-CoS$\mathrm{FM}^{\mathrm{b}}$ ), i.e., the cone axis $\boldsymbol{l}$ is still along the $\mathrm{b}$ axis, while the angle between the magnetic moment and the $\boldsymbol{l}$ (b axis) reduces to $70^{\circ}$ (Fig.2a(Fig.S12), Table S2). Meanwhile, the moment remains nearly unchanged with $\mu_{(\mathrm{Mn} / \mathrm{Fe})}=3.04(3) \mu_{\mathrm{B}}$. Such a $70^{\circ}-\mathrm{CoS}-\mathrm{FM}^{\mathrm{b}}$ persists the single spin configuration until $\mathrm{H}=1 \mathrm{~T}$. As the $\mathrm{H}>1 \mathrm{~T}$, a linear FM structure (with the moment $\left(\mathrm{M}_{(\mathrm{Mn} / \mathrm{Fe})}=3.08(6) \mu_{\mathrm{B}}\right)$ still unchanged but aligning $\mathrm{b}$ axis) begin to form, and its fraction gradually increase with increasing $\mathrm{H}$. At the $\mathrm{H}$ reaches $5 \mathrm{~T}$, the $70^{\circ}$-CoS-FM ${ }^{\mathrm{b}}$ totally evolves into the linear FM structure (Fig.2a(Fig.S12), 
Table S2). One can note that the magnetic moment $\mathrm{M}$ at $\mathrm{Mn}(\mathrm{Fe})$ sites remains nearly constant $\left(3.06 \mu_{\mathrm{B}}\right)$ through $0 \leq \mathrm{H} \leq 5 \mathrm{~T}$ (Fig.S13a). Further refinements indicated that the variation of Mn-Mn distances in the entire magnetic field range $0 \leq \mathrm{H} \leq 5 \mathrm{~T}$ is less than $0.2 \AA$ (Fig.S13b), which can convert the spin-structure, but may be not enough to change the atomic moment.

To know more about the evolution process of the CyS-AFM ${ }^{\mathrm{b}}$ spin-structure with magnetic field, we further monitored the $(000)^{ \pm}$magnetic satellite with continuously increasing magnetic field, as shown in Fig.S14. One can note that the peak intensity of $(000)^{ \pm}$magnetic satellite shows a step-like increase around $0.6 \mathrm{~T}$. But as the $\mathrm{H}>1 \mathrm{~T}$, the peak intensity of $(000)^{ \pm}$magnetic satellite becomes decreasing almost linearly with magnetic field, and nearly disappears at $4 \mathrm{~T}$, indicating the gradual reduction of the incommensurate $70^{\circ}-\mathrm{CoS}-\mathrm{FM}^{\mathrm{b}}$ spin structure with increasing H. Simultaneously, gradual enhancements of several orthorhombic crystal reflections can be identified in the NPD patterns as $\mathrm{H}>1 \mathrm{~T}$, such as the typical (112) and (103) peaks (upper panel of Fig.S10, Fig.S14), indicating the appearance and gradual growth of the linear ferromagnetic reflections with increasing magnetic field. As mentioned by Shull et al ${ }^{[\mathrm{S} 19]}$, when the magnetic moments are aligned within the domains, the magnetic scattering will appear in the Bragg peaks together with the nuclear scattering, i.e. the magnetic scattering overlaps with the nuclear scattering.

In the refinement of magnetic structure, the most important parameter is the scale factor (S), which is a function of the magnetic form factor (mainly related to the parameters used to describe the magnetic structure, such as the spherical coordinate parameters) and the magnitude of the magnetic moment. During refinement, the scale factor of the magnetic structure should be kept constant and equal to the scale factor obtained from the corresponding nuclear structure (the scale factor of a nuclear structure is easy to get), otherwise the magnetic structure and the magnetic moment amplitudes cannot be properly determined. If the scale factor of a magnetic/structure phase $\mathrm{i}\left(\mathrm{S}_{\mathrm{i}}\right)$ is determined, we can obtain the weight fractions, $\mathrm{W}_{\mathrm{i}}$, by

$$
W_{i}=\frac{s_{i} \cdot m_{i}}{\sum_{1}^{n} S_{j} \cdot m_{j}}
$$

where $m_{i}$ is the unit cell mass for the phase $i$.

For the systems with multiple magnetic structures, if the different magnetic structures correspond to the different crystal structures, the weight fractions of the magnetic structures are relatively easy to be refined out. However, there is no any split for the crystal reflections during the spin structure evolution from the $70^{\circ}-\mathrm{CoS}-\mathrm{FM}^{\mathrm{b}}$ to the linear FM, which suggest that the two magnetic structures correspond to the same orthorhombic (Pnma) structure.

In this situation, the sum of the scale factor of $70^{\circ}-\mathrm{CoS}-\mathrm{FM}^{\mathrm{b}}$ and linear FM should be equal to the scale factor of the orthorhombic structure, i.e. $\mathrm{S}_{\mathrm{Orth}}=\mathrm{S}_{70^{\circ}-\mathrm{CoS}-\mathrm{FM}}{ }^{\mathrm{b}}+\mathrm{S}_{\text {linear FM. }}$. As mentioned above, the scale factor is a function of the magnetic form factor and the magnitude of the magnetic moment. To calculate the true weight fractions of these two magnetic structures via function (3), the magnetic form factor and the magnetic moment need to be fixed to exclude their influence. This is reasonable noting that the two end magnetic structures, i.e. $70^{\circ}-\mathrm{CoS}-\mathrm{FM}^{\mathrm{b}}$ and linear $\mathrm{FM}$, and the corresponding moment M (magnetic form factor) have been determined in their clean phase by NPD under $0.6 \mathrm{~T}$ and $5 \mathrm{~T}$, respectively. In other words, the magnetic moment at $\mathrm{Mn}(\mathrm{Fe})$ sites remains nearly constant $\left(3.06 \mu_{\mathrm{B}}\right)$ through $0 \leq \mathrm{H} \leq 5 \mathrm{~T}$ (Fig.S13a). Eventually, we got the magnetic phase fraction evolution with $\mathrm{H}$, as shown in Table S2. 


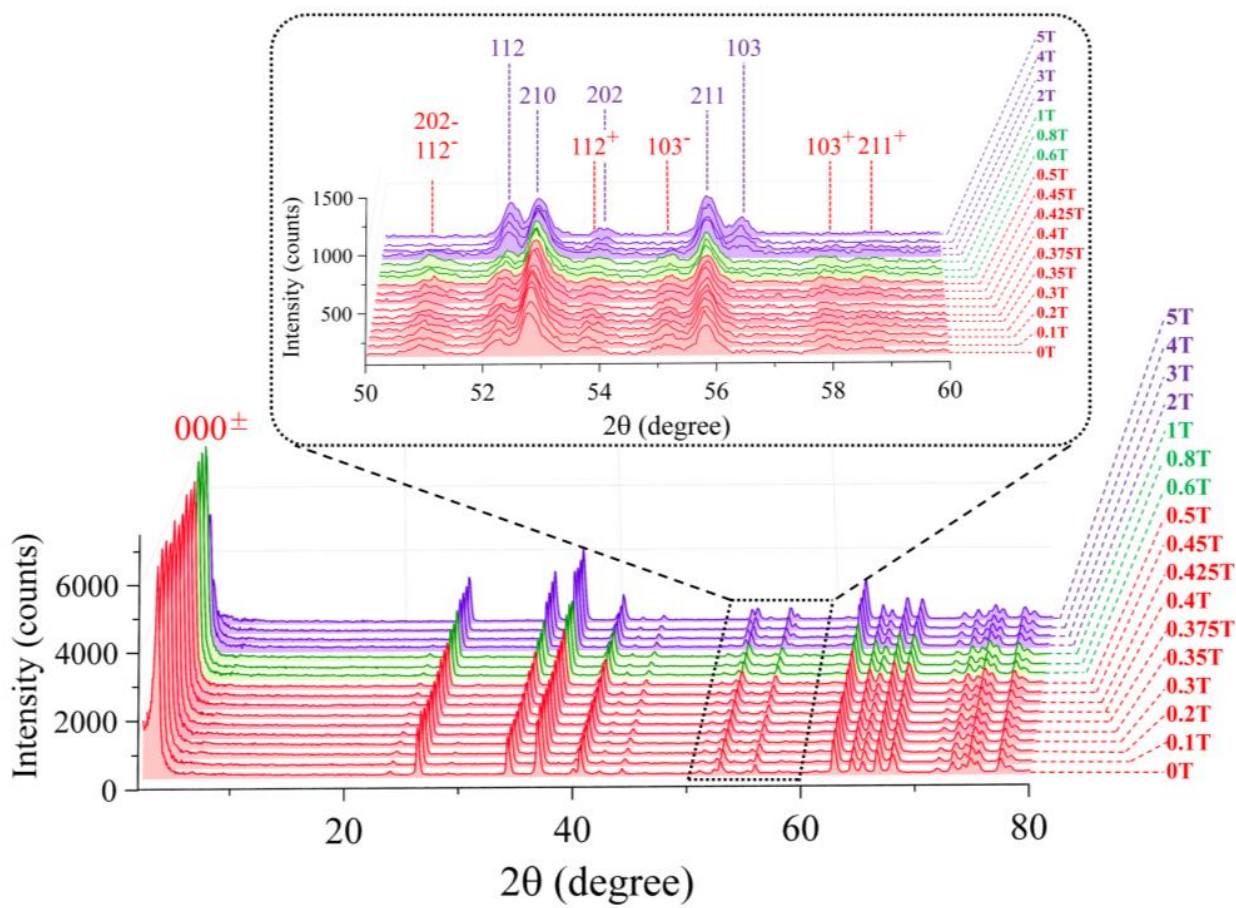

Fig.S10 Variable magnetic field NPD patterns at 5K. Up panel: the specific NPD patterns in $50^{\circ}-60^{\circ}$, where the incommensurate magnetic satellites (red) and orthorhombic crystal reflections (purple) are indexed.

Table S2 NPD refinement results under variable magnetic fields $(\mathrm{H})$. Magnetic moment $\mathrm{M}$, magnetic structure (cycloidal spiral abbreviated as CyS, conical spiral abbreviated as CoS, FM refers to linear FM) and the corresponding parameters $(\theta, \varphi, \psi)$ for the spiral magnetic structure, and the phase fraction of linear FM phase under different magnetic fields at $5 \mathrm{~K}$. Refined weighted profile $\mathrm{R}$-factor $\mathrm{R}_{\mathrm{wp}}$ and $\chi^{2}$ are also listed.

\begin{tabular}{|c|c|c|c|c|c|c|}
\hline $\mathrm{H}(\mathrm{T})$ & $\mathrm{M}\left(\mu_{\mathrm{B}}\right)$ & Magn. Stru. & $\theta \& \varphi$ & $\psi$ & $\begin{array}{c}\text { Phase fraction of } \\
\text { linear FM phase } \\
(\%)\end{array}$ & $\mathrm{R}_{\mathrm{wp}}, \chi^{2}$ \\
\hline 0 & $3.06(3)$ & CyS-AFM ${ }^{b}$ & $90^{\circ}, 90^{\circ}$ & $90^{\circ}$ & 0 & $8.81,1.27$ \\
\hline 0.1 & $3.05(3)$ & CyS-AFM ${ }^{b}$ & $90^{\circ}, 90^{\circ}$ & $90^{\circ}$ & 0 & $8.63,1.30$ \\
\hline 0.2 & $3.05(3)$ & CyS-AFM ${ }^{b}$ & $90^{\circ}, 90^{\circ}$ & $90^{\circ}$ & 0 & $8.75,1.33$ \\
\hline 0.3 & $3.06(3)$ & CyS-AFM ${ }^{b}$ & $90^{\circ}, 90^{\circ}$ & $90^{\circ}$ & 0 & $9.07,1.38$ \\
\hline 0.35 & $3.04(3)$ & CyS-AFM ${ }^{b}$ & $90^{\circ}, 90^{\circ}$ & $90^{\circ}$ & 0 & $8.66,1.31$ \\
\hline 0.375 & $3.07(3)$ & CyS-AFM ${ }^{b}$ & $90^{\circ}, 90^{\circ}$ & $90^{\circ}$ & 0 & $9.59,1.50$ \\
\hline 0.4 & $3.07(3)$ & CyS-AFM ${ }^{b}$ & $90^{\circ}, 90^{\circ}$ & $90^{\circ}$ & 0 & $9.59,1.50$ \\
\hline 0.425 & $3.05(3)$ & CyS-AFM ${ }^{b}$ & $90^{\circ}, 90^{\circ}$ & $90^{\circ}$ & 0 & $9.20,1.48$ \\
\hline 0.45 & $3.06(3)$ & CyS-AFM ${ }^{b}$ & $90^{\circ}, 90^{\circ}$ & $90^{\circ}$ & 0 & $8.78,1.33$ \\
\hline 0.5 & $3.05(3)$ & CyS-AFM ${ }^{b}$ & $90^{\circ}, 90^{\circ}$ & $90^{\circ}$ & 0 & $8.76,1.35$ \\
\hline 0.6 & $3.07(3)$ & $70^{\circ}-\mathrm{CoS}-\mathrm{FM}^{\mathrm{b}}$ & $90^{\circ}, 90^{\circ}$ & $70^{\circ}$ & 0 & $8.79,1.42$ \\
\hline 0.8 & $3.04(3)$ & $70^{\circ}-$ CoS-FM $^{b}$ & $90^{\circ}, 90^{\circ}$ & $70^{\circ}$ & 0 & $8.90,1.33$ \\
\hline 1 & $3.07(3)$ & $70^{\circ}-C_{0 S}-F^{b}$ & $90^{\circ}, 90^{\circ}$ & $70^{\circ}$ & 0 & $9.00,1.47$ \\
\hline 2 & 3.06 & $70^{\circ}-\mathrm{CoS}-\mathrm{FM}^{\mathrm{b}} \& \mathrm{FM}$ & $90^{\circ}, 90^{\circ}$ & $70^{\circ}$ & 45.6 & $9.10,1.50$ \\
\hline 3 & 3.06 & $70^{\circ}-\mathrm{CoS}-\mathrm{FM}^{\mathrm{b}} \& \mathrm{FM}$ & $90^{\circ}, 90^{\circ}$ & $70^{\circ}$ & 76.5 & $9.39,1.40$ \\
\hline 4 & 3.06 & $70^{\circ}-\mathrm{CoS}-\mathrm{FM}^{\mathrm{b}} \& \mathrm{FM}$ & $90^{\circ}, 90^{\circ}$ & $70^{\circ}$ & 93.5 & $9.37,1.38$ \\
\hline 5 & $3.08(5)$ & FM & -- & -- & 100 & $9.19,1.34$ \\
\hline
\end{tabular}



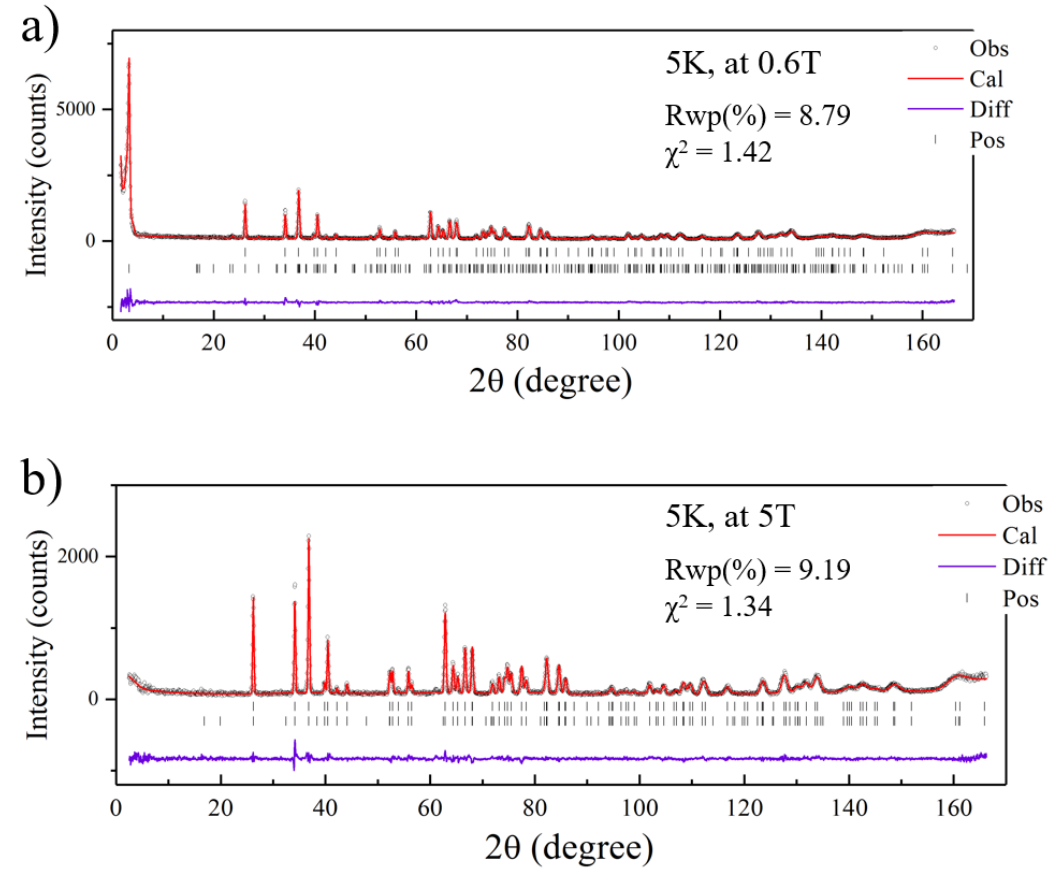

Fig.S11 Observed (black circle), calculated (red line) NPD patterns, their difference (purple line), peak position (black bar) for $\mathrm{Mn}_{0.87} \mathrm{Fe}_{0.13} \mathrm{NiGe}$ collected at $5 \mathrm{~K}$ and under a) $0.6 \mathrm{~T}$ and b) $5 \mathrm{~T}$, respectively.

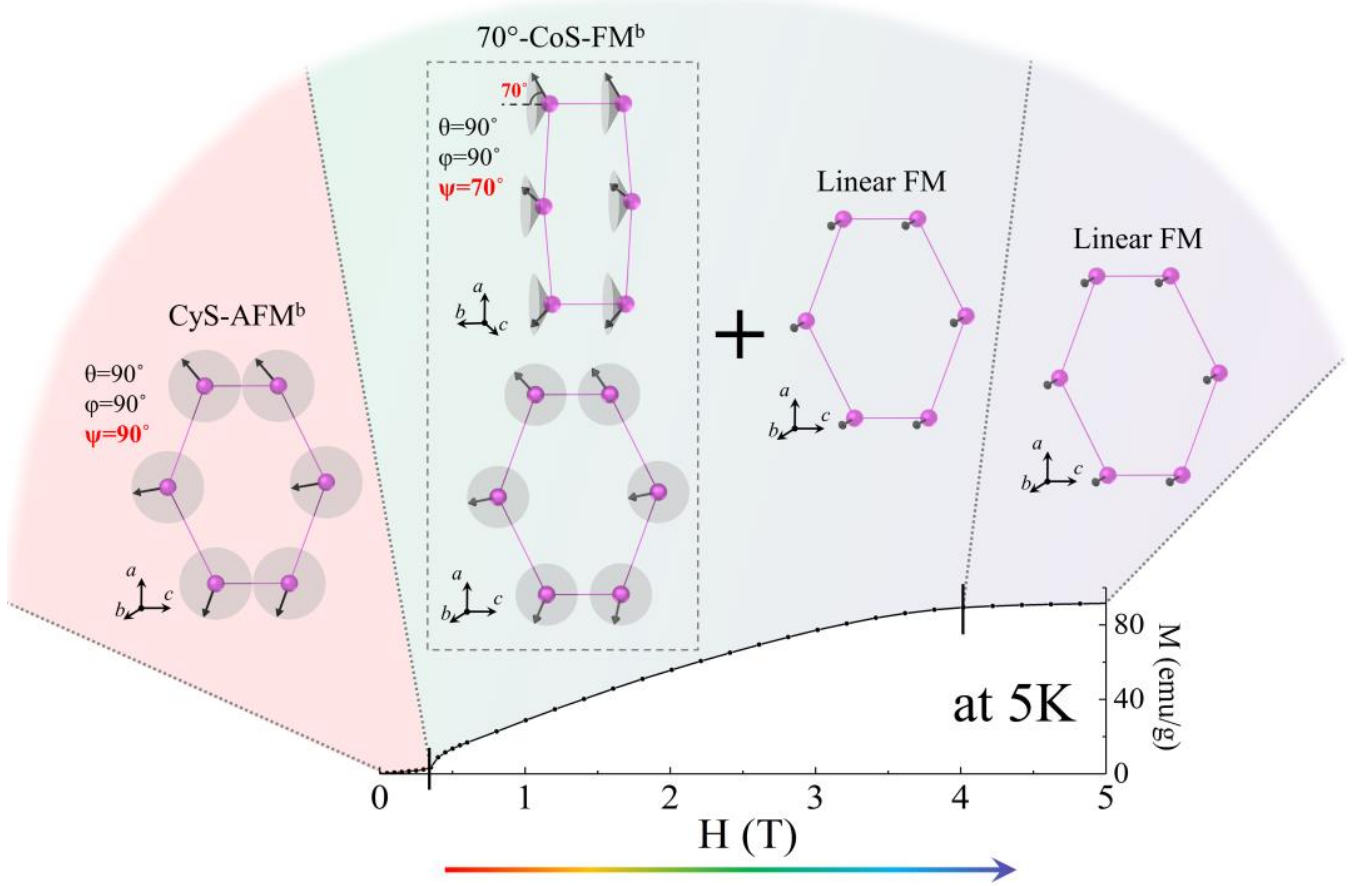

Fig.S12 M-H curve at 5K with the sketch of spin structure evolution with magnetic field. 

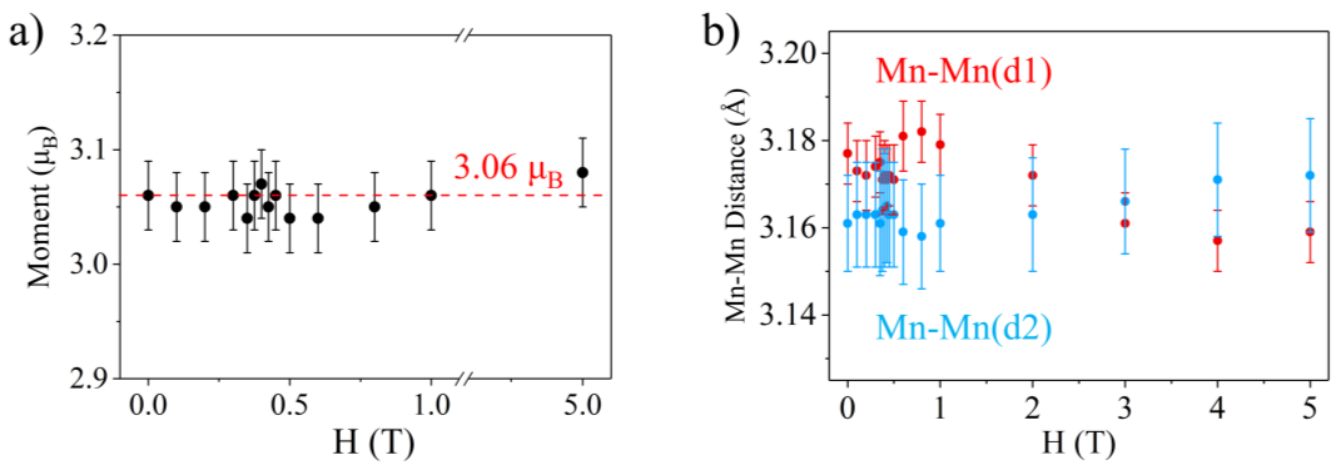

Fig.S13 Variation of a) the refined magnetic moment at $\mathrm{Mn}(\mathrm{Fe})$ sites and b) the $\mathrm{Mn}-\mathrm{Mn}$ distances with magnetic field $\mathrm{H}$ at $5 \mathrm{~K}$.

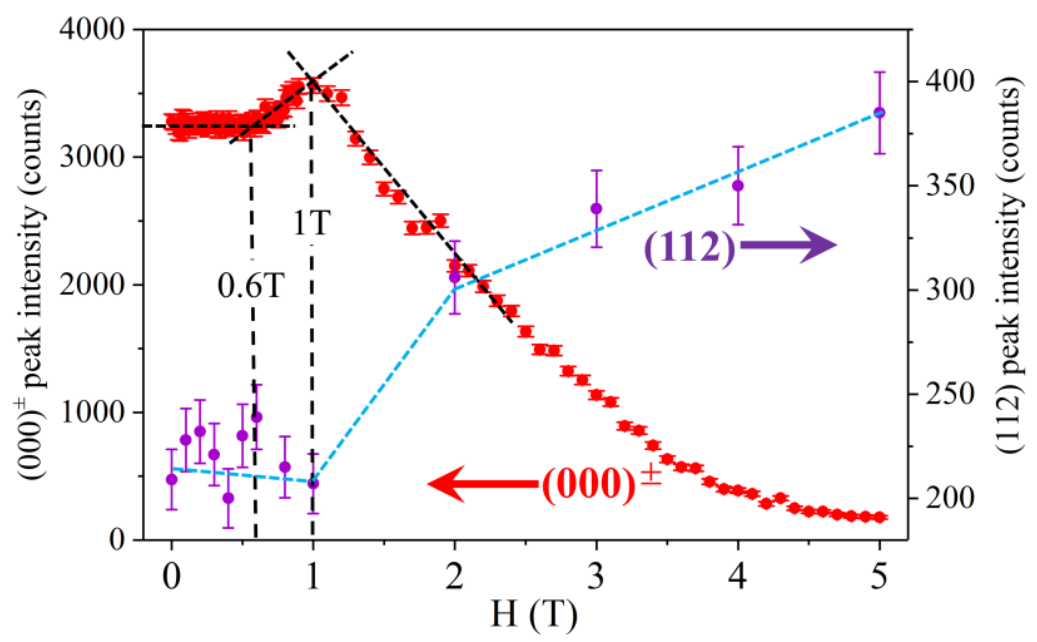

Fig.S14 The peak intensity of the $(000)^{ \pm}$magnetic satellite and the (112) crystal reflection as a function of magnetic field at $5 \mathrm{~K}$.

The $70^{\circ}-\mathrm{CoS}-\mathrm{FM}^{\mathrm{a}}$ and $70^{\circ}-\mathrm{CoS}-\mathrm{FM}^{\mathrm{b}}$ are both the conical spiral spin structure with the same $70^{\circ}$ angle between the magnetic moment and the cone axis, but the direction of their cone axis is different. The cone axis of $70^{\circ}-\mathrm{CoS}-\mathrm{FM}^{\mathrm{a}}$ is along the a-axis, while the $70^{\circ}-\mathrm{CoS}-\mathrm{FM}^{\mathrm{a}}$ is along the baxis. As a result, their spin direction is totally different.

In an NPD pattern, the magnetic peak intensity is expressed as $I=C\left|F_{h k l}\right|^{2}$, where the $F_{h k l}$ is the scattering factor. For the magnetic reflections, the scattering factor $F_{h k l}=$ $\sum q_{j} f_{M j} \exp [2 \pi i(h x+k y+l z)] e^{-2 w}$, where $q_{j}$ and $f_{M j}$ are the magnetic interaction vector and the magnetic form factor for atom $j$, respectively, and $\mathrm{w}$ is the Debye Waller factor ${ }^{[\mathrm{S} 20]}$. Among them, the magnetic interaction vector $q$ is highly dependent on the spin direction as shown in Fig.S15. The magnetic interaction vector $q$ is always perpendicular to the scattering vector, and its module $|q|=\sin \alpha$, where $\alpha$ is the angle between the neutron scattering vector $\varepsilon$ and magnetic moment unit $\mathrm{K}$. Therefore, the magnetic peak intensity is also dependent on the spin direction.

Since the spin directions of $70^{\circ}-\mathrm{CoS}-\mathrm{FM}^{\mathrm{a}}$ and $70^{\circ}-\mathrm{CoS}-\mathrm{FM}^{\mathrm{b}}$ are totally different, their magnetic peak intensities are also different, especially for the $(000)^{ \pm}$magnetic satellite as shown in Fig.S16. 


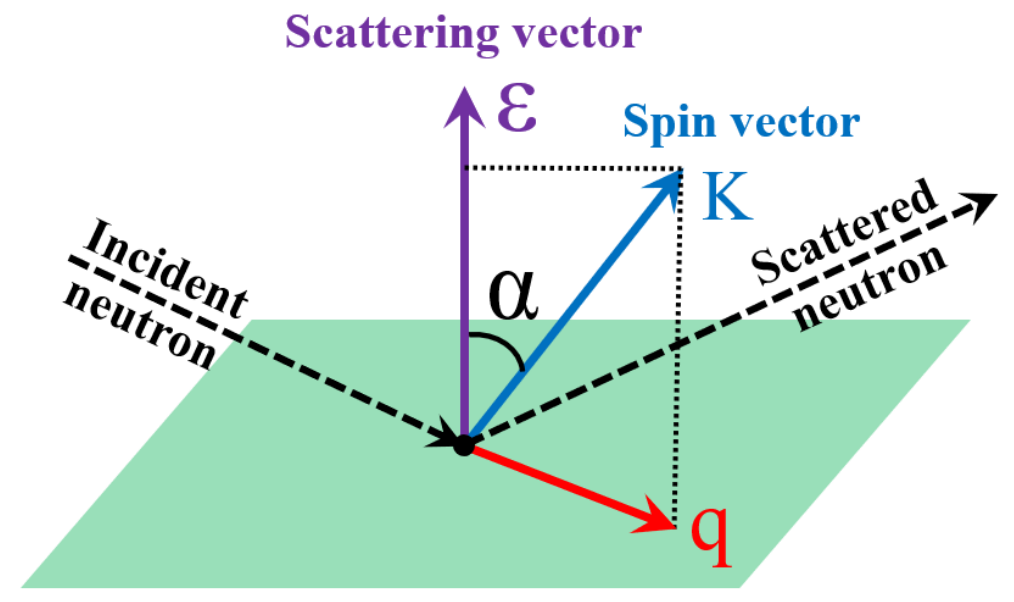

Fig.S15 Definition of the vectors relevant in the evaluation of the magnetic structure factor. $\varepsilon$ and $\mathrm{K}$ are unit vectors in the directions of the neutron scattering and spin, respectively.

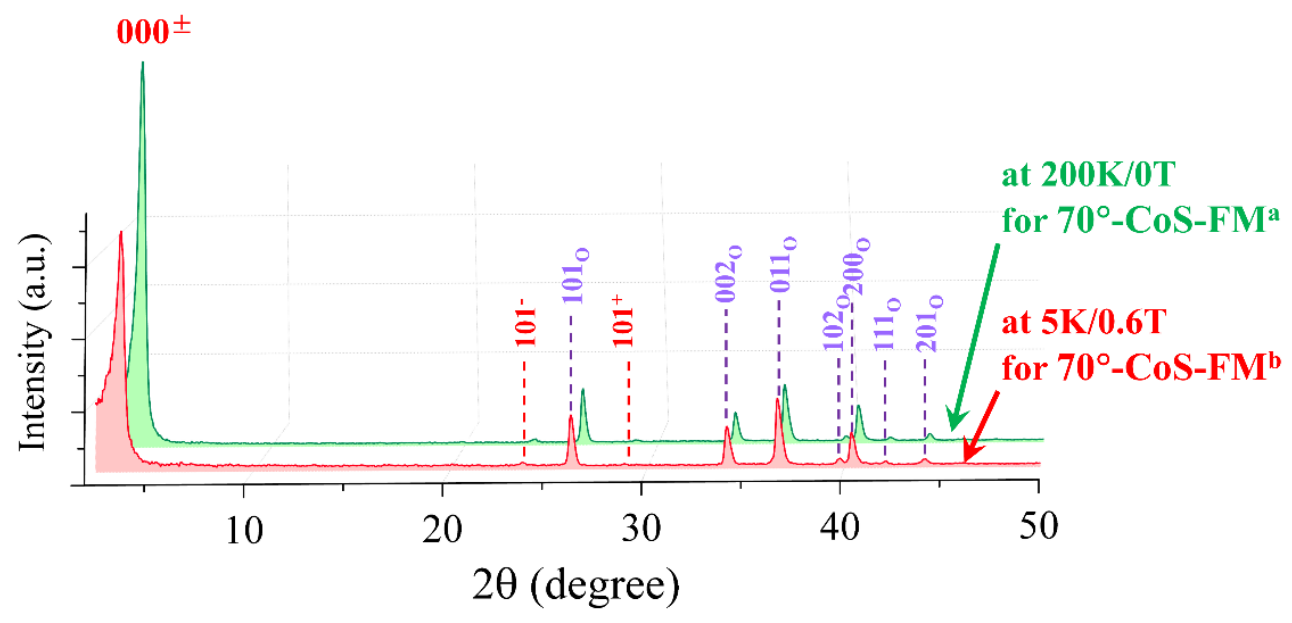

Fig.S16 The NPD patterns at 5K/0.6T (in $70^{\circ}-\mathrm{CoS}-\mathrm{FM}^{\mathrm{b}}$ region) and at $200 \mathrm{~K} / 0 \mathrm{~T}$ (in $70^{\circ}-\mathrm{CoS}-\mathrm{FM}^{\mathrm{a}}$ region). For comparison, the peak intensities are normalized based on the orthorhombic (101) crystal reflection.

To know more about the evolution process of spin structure with $\mathrm{H}$ at higher temperatures in the CyS-AFM ${ }^{\mathrm{b}}$ region, the $(000)^{ \pm}$magnetic satellite as a function of magnetic field at $80 \mathrm{~K}$ and $130 \mathrm{~K}$ were also monitored, as shown in the up panel of Fig.S17. The performances of the $(000)^{ \pm}$peak with magnetic field are similar to that at $5 \mathrm{~K}$. But, the critical magnetic field, $\mathrm{H}_{\mathrm{C}}$, for the spin structure conversion from the CyS-AFM ${ }^{\mathrm{b}}$ to $70^{\circ}-\mathrm{CoS}-\mathrm{FM}^{\mathrm{b}}$ decreases from $0.6 \mathrm{~T}(5 \mathrm{~K})$ to $0.5 \mathrm{~T}$ at $80 \mathrm{~K}$ and $0.2 \mathrm{~T}$ at $130 \mathrm{~K}$. Besides, the initial magnetic field for the spin structure conversion from the $70^{\circ}-\mathrm{CoS}-\mathrm{FM}^{\mathrm{b}}$ to the linear FM also decreases from $1 \mathrm{~T}(5 \mathrm{~K})$ to $0.95 \mathrm{~T}$ at $80 \mathrm{~K}$ and $0.5 \mathrm{~T}$ at $130 \mathrm{~K}$.

For the $70^{\circ}-\mathrm{CoS}-\mathrm{FM}^{\mathrm{a}}$ region, the $(000)^{ \pm}$magnetic satellite as a function of magnetic field at $200 \mathrm{~K}$ was also monitored, as shown in the down panel of Fig.S17. The $(000)^{ \pm}$peak shows almost monotonous decrease with increasing magnetic field, and eventually disappears at around $2 \mathrm{~T}$. It suggests that the $70^{\circ}$-CoS-FM ${ }^{\mathrm{a}}$ gradually transforms to the linear FM with external magnetic field applied, and the transition completes at around 2T. Combining with the macroscopic magnetic measurements (M-H curves) (SI-9, Fig.S19), the phase diagram under variable magnetic field and temperature was constructed, as shown in Fig.2c.

The deviation of $\mathrm{H}_{\mathrm{C}}$ obtained from NPD compared with the one from M-H curves can be 
understandable considering the different equipment providing magnetic field environments and the possible differences from sample to sample.

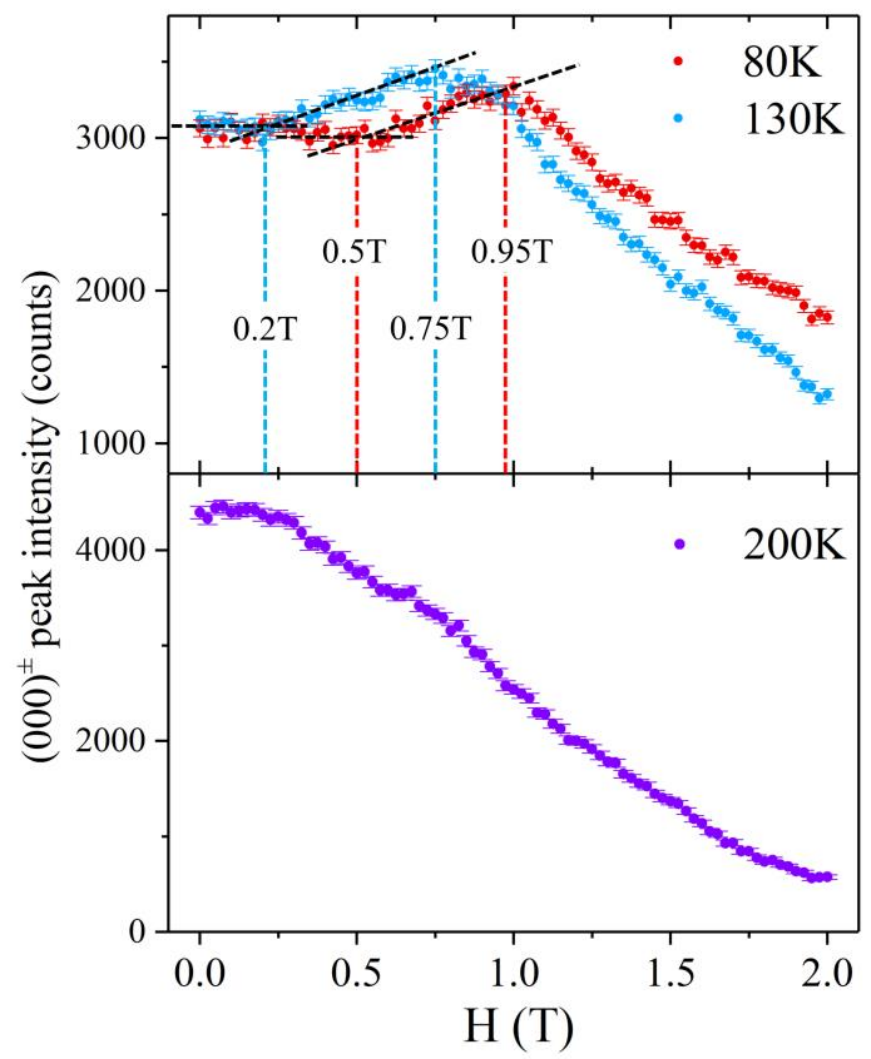

Fig.S17 The peak intensity of the $(000)^{ \pm}$magnetic satellite as a function of magnetic fields at $80 \mathrm{~K}$ (red dots), $130 \mathrm{~K}$ (blue dots) and 200K (purple dots), respectively.

\section{SI-9 Calculation for $\Delta M_{H P}$ (BME) and baromagnetic coefficient (BMC) $d$}

Fig.3a displays the $\mathrm{M}-\mathrm{H}$ curves measured under variable pressures at different temperatures. At the CyS-AFM ${ }^{\mathrm{b}}$ region $(\mathrm{T} \leq 150 \mathrm{~K}), \mathrm{P} \sim 1.8 \mathrm{kbar}$ pressure cannot remarkably alter the magnetizing process. The shape of $\mathrm{H}-\mathrm{M}$ curves persists almost the same while the saturated moment remains unchanged, except a slight reduction of saturated magnetic field (Hs). Meanwhile, the critical field $\mathrm{Hc}$ triggering the transition from $\mathrm{CyS}-\mathrm{AFM}^{\mathrm{b}}$ to $70^{\circ}-\mathrm{CoS}-\mathrm{FM}^{\mathrm{b}}$ also keeps unchanged at the same temperature.

Further increasing pressure up to $6 \mathrm{kbar}$ makes the magnetizing process completely different from the situation at atmospheric pressure (Fig.3a-(1-5)). The isothermal M-H curves at $\mathrm{T} \leq 150 \mathrm{~K}$ become easily magnetized particularly at low field region $\mathrm{H} \leq 0.5 \mathrm{~T}$, similar to the spontaneous magnetizing behaviour of a typical FM magnet. More importantly, the saturated moment notably reduces, as shown in Table S3. The reduction ratio of saturated moment increases with temperature, i.e. $22.3 \%$ for $5 \mathrm{~K}, 24.7 \%$ for $100 \mathrm{~K}, 26.9 \%$ for $120 \mathrm{~K}, 33.4 \%$ for $150 \mathrm{~K}$. Continuously increasing pressure to $8.3 \mathrm{kbar}$ leads to further easier magnetized and further reduction of saturated moment (Table S3), while the shape of $\mathrm{M}-\mathrm{H}$ curves does not change too much (Fig.3a-(1-5)).

Moreover, we performed the isothermal magnetization measurements (M-H curves) with full loop on ascending and descending processes. Typically, Fig.S18 shows the M-H curves under 4 continuously varying hydrostatic pressures $(0 \mathrm{kbar}, 1.8 \mathrm{kbar}, 6.0 \mathrm{kbar}, 8.3 \mathrm{kbar})$ at $5 \mathrm{~K}$. One can find that the two branches of $\mathrm{M}-\mathrm{H}$ curve on ascending and descending process almost coincide for either 
the CyS-AFM ${ }^{\mathrm{b}}$ case under pressures $\mathrm{P} \leqslant 1.8 \mathrm{kbar}$ or the $45^{\circ}-\mathrm{CoS}-\mathrm{FM}^{\mathrm{a}}$ state generated by higher pressure. At first glance, the two branches on rising and falling processes cannot even be distinguished. For details, the enlarged areas around the metamagnetic transition and initial magnetization process are presented on right and upper panels of Fig.R3, respectively. These results indicate that the magnetizing process across the metamagnetic transition is completely reversible with almost zero hysteresis for CyS-AFM ${ }^{\mathrm{b}}$ state, while the magnetizing process is also reversible for $45^{\circ}-$ CoS-FM ${ }^{\mathrm{a}}$ state.
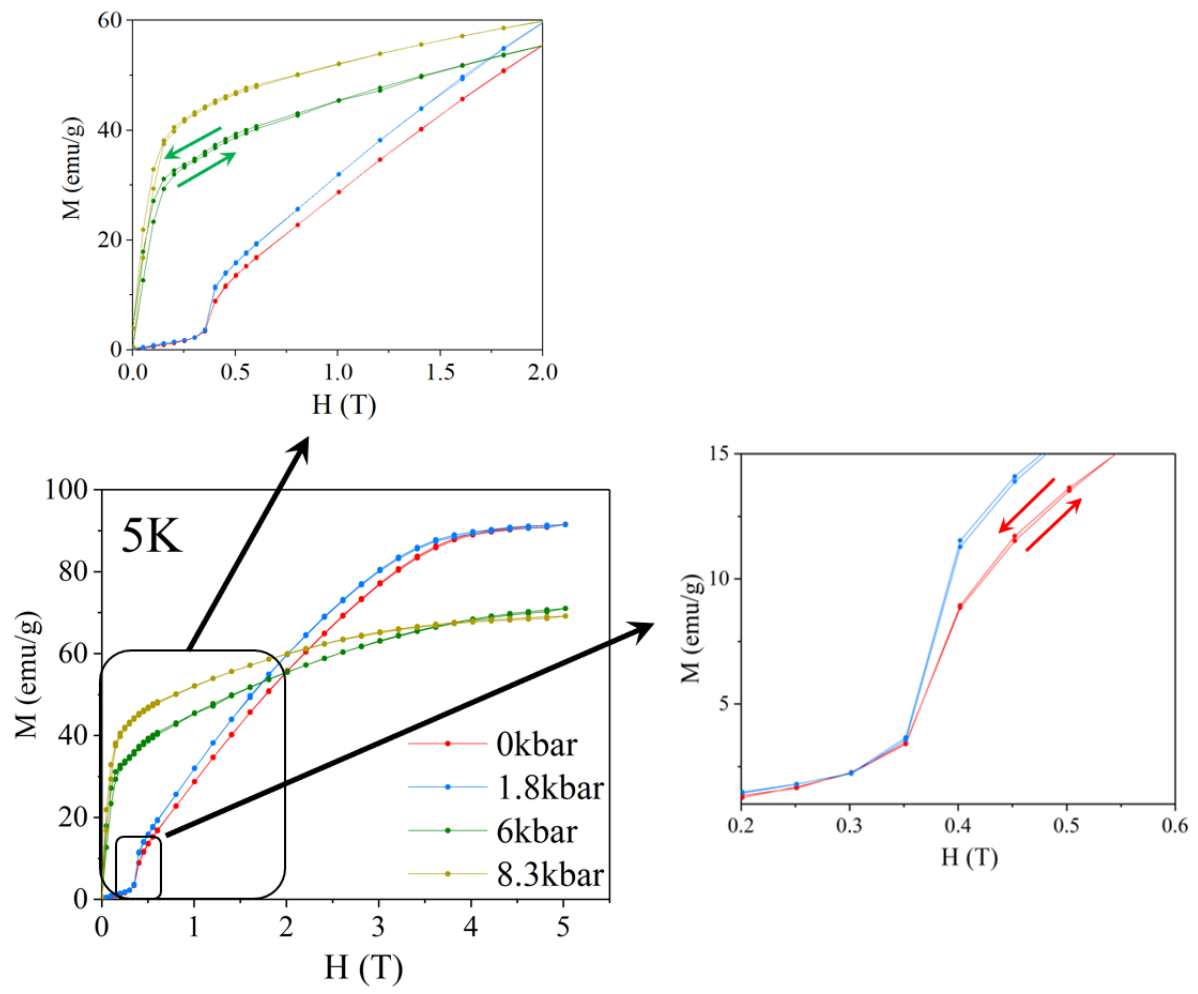

Fig.S18 Isothermal magnetization measurements (M-H curves) on ascending and descending processes under different hydrostatic pressures at $5 \mathrm{~K}$. Right and upper panels show the enlarged details around the metamagnetic transition and initial magnetization process, respectively.

Table S3 The saturated moment $\left(\mathrm{M}_{\mathrm{S}}\right)$ from isothermal M-H curves measured under variable pressures at different temperatures, and the magnetic moment on $\mathrm{Mn} / \mathrm{Fe}$ sites $\left(\mathrm{M}_{(\mathrm{Mn} / \mathrm{Fe})}\right)$ from the NPD refinements under variable temperatures and pressure. (atmospheric pressure is labeled as $0 \mathrm{kbar}$ )

\begin{tabular}{|c|c|c|c|c|c|}
\hline \multirow{6}{*}{$\mathrm{Ms}_{\mathrm{s}}\left(\mu_{\mathrm{B}}\right)$ from $\mathrm{M}-\mathrm{H}$} & & $0 \mathrm{kbar}$ & $1.8 \mathrm{kbar}$ & $6.0 \mathrm{kbar}$ & $8.3 \mathrm{kbar}$ \\
\hline & $5 \mathrm{~K}$ & 3.05 & 3.05 & 2.37 & 2.31 \\
\hline & $100 \mathrm{~K}$ & 2.87 & 2.87 & 2.16 & 2.05 \\
\hline & $120 \mathrm{~K}$ & 2.83 & 2.83 & 2.07 & 1.94 \\
\hline & $150 \mathrm{~K}$ & 2.78 & 2.78 & 1.85 & 1.66 \\
\hline & $200 \mathrm{~K}$ & 2.64 & 2.64 & - & - \\
\hline \multirow{5}{*}{$\mathrm{M}_{(\mathrm{Mn} / \mathrm{Fe})}\left(\mu_{\mathrm{B}}\right)$ from NPD } & & $0 \mathrm{kbar}$ & $2.0 \mathrm{kbar}$ & $6.0 \mathrm{kbar}$ & \\
\hline & $5 \mathrm{~K}$ & $3.06(2)$ & - & - & - \\
\hline & $100 \mathrm{~K}$ & $2.89(2)$ & - & - & - \\
\hline & $120 \mathrm{~K}$ & $2.80(3)$ & $2.80(3)$ & $2.08(2)$ & - \\
\hline & $200 \mathrm{~K}$ & $2.66(1)$ & - & - & - \\
\hline
\end{tabular}


The distinct magnetizing behaviour at $\mathrm{P}=0 \mathrm{kbar}$ and $\mathrm{P} \geq 6 \mathrm{kbar}$ leads to the occurrence of large BME. Based on the M-H curves (Fig.3a-(1-5)) and equations (1) and (2) in SI-1 above, $\Delta \mathrm{M}_{\mathrm{HP}}$ was calculated to quantify the BME, and further calculate the corresponding BMC to quantify the sensitivity as a sensor. The $\Delta \mathrm{M}_{\mathrm{HP}}$ reaches extremes at the saturated magnetic field $\mathrm{Hs}$ and also at a low critical magnetic field $\mathrm{H}_{\mathrm{C}}$ triggering the conversion from $\mathrm{CyS}-\mathrm{AFM}^{\mathrm{b}}$ to $70^{\circ}-\mathrm{CoS}-\mathrm{FM}^{\mathrm{b}}$, as shown in Fig.3a-(6-10), while the sign of BME changes. At low magnetic field, positive BME appears below $150 \mathrm{~K}$, which peaks exactly around the Hc. Note that increasing temperature from $5 \mathrm{~K}$ to $150 \mathrm{~K}$ causes the Hc monotonous decrease from 0.35T to 0 (Fig.S19). Typically, the BME under a low $\mathrm{H}=0.35 \mathrm{~T}$ reaches +32.0 and $+40.5 \mathrm{emu} / \mathrm{g}$ (Fig.S20), while the corresponding BMC reaches $d \sim 5.34$ and $\sim 4.88 \mathrm{emu} \cdot \mathrm{g}^{-1} \mathrm{kbar}^{-1}$, under $6.0 \mathrm{kbar}$ and $8.3 \mathrm{kbar}$, respectively. With increasing $\mathrm{H}$, the sign of BME turns to be negative, and the BME reaches the negative maximum at around the Hs and then keeps almost unchanged with further increasing $\mathrm{H}$ (Fig.3a-(6-10)). As the temperature increasing from $5 \mathrm{~K}$ to $200 \mathrm{~K}$, the Hs monotonous decrease from $4 \mathrm{~T}$ to $2.2 \mathrm{~T}$ (Fig.S19). It means the working magnetic field where the BME peaks gradually decreases with temperature rising. Note that the negative $\mathrm{BME}$ at $5 \mathrm{~K}$ and $200 \mathrm{~K}$ reaches the maximum at $\mathrm{Hs} \sim 4 \mathrm{~T}$ and $2.2 \mathrm{~T}$, while the magnitude under $6.0 \mathrm{kbar}$ and $8.3 \mathrm{kbar}$ is -20.9 and $-21.4 \mathrm{emu} / \mathrm{g}(5 \mathrm{~K}),-54.2$ and $-62.7 \mathrm{emu} / \mathrm{g}(200 \mathrm{~K})$, and corresponding BMC is $\mathrm{d} \sim 3.49$ and $2.57(5 \mathrm{~K}), 9.03$ and $7.55 \mathrm{emu} \cdot \mathrm{g}^{-1} \cdot \mathrm{kbar}^{-1}(200 \mathrm{~K})$, respectively (Fig.S20).

Obviously, the BME in $\mathrm{Mn}_{0.87} \mathrm{Fe}_{0.13} \mathrm{NiGe}$ is more attractive compared to the $\mathrm{Mn}_{3} \mathrm{Ga}_{0.95} \mathrm{~N}_{0.94}$ previously reported. ${ }^{[\mathrm{S} 1]}$ In $\mathrm{Mn}_{3} \mathrm{Ga}_{0.95} \mathrm{~N}_{0.94}$, the maximal $\Delta \mathrm{M}_{\mathrm{HP}}$ is $+14.4 \mathrm{emu} / \mathrm{g}$ at $130 \mathrm{~K} / 7.5 \mathrm{kbar}$ under 1T-5T magnetic field, equivalent BMC is $d \sim 1.92 \mathrm{emu} \cdot \mathrm{g} \mathrm{g}^{-1} \mathrm{kbar}^{-1}$ with a temperature window $115 \mathrm{~K}$ (75-190K). In present $\mathrm{Mn}_{0.87} \mathrm{Fe}_{0.13} \mathrm{NiGe}$, the maximal $\mathrm{BMC} \mathrm{d}=9.03 \mathrm{emu} \cdot \mathrm{g}^{-1} \mathrm{kbar}^{-1}$ at $2.2 \mathrm{~T}$ is 4.7 times larger than that of $\mathrm{Mn}_{3} \mathrm{Ga}_{0.95} \mathrm{~N}_{0.94}$, and the temperature range $(0-250 \mathrm{~K})$ is also significantly wider (Fig.3f).

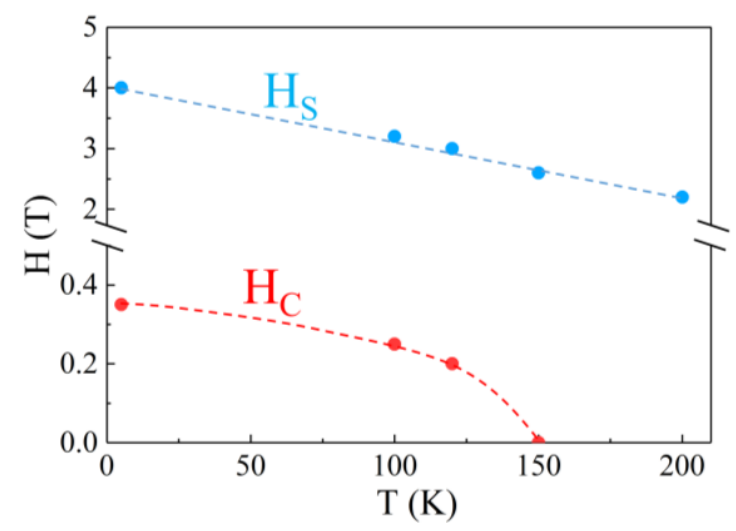

Fig.S19 The critical magnetic field Hc and the saturated magnetic field $\mathrm{Hs}$ (obtained from $\mathrm{M}-\mathrm{H}$ curves) as a function of temperature. 

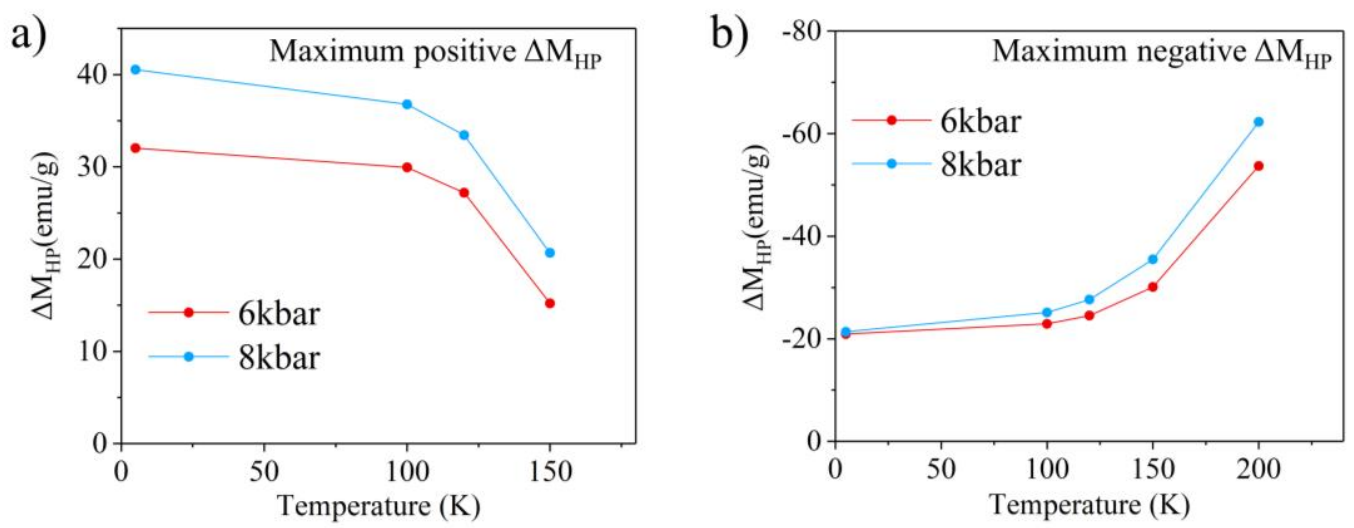

Fig.S20 Variation of the maximum $\Delta \mathrm{M}_{\mathrm{HP}}$ with temperature and pressure, under a) a low magnetic field $(\mathrm{Hc} \leq 0.35 \mathrm{~T})$ and b) high magnetic field $(2 \mathrm{~T} \leq \mathrm{H} \leq 5 \mathrm{~T})$, respectively.

\section{SI-10 NPD measurements under variable pressure}

Fig.S21 shows the NPD patterns collected under 4 continuously varying pressures ( $0 \mathrm{kbar}, 2 \mathrm{kbar}$, $4 \mathrm{kbar}, 6 \mathrm{kbar}$ ) at $120 \mathrm{~K}$ in the CyS-AFM ${ }^{\mathrm{b}}$ region. The spectra quantities are not as good as those under magnetic fields due to the influence of pressure cell, but the incommensurate magnetic satellites besides $(000)^{ \pm}$can be still identified clearly (marked by red, inset of Fig.S21). Since the pressure cell made of $\mathrm{Al}$ was used, there are $\mathrm{Al}$ diffraction peaks (black) and minor other unknown impurity peaks (black, possibly from device environments) appearing in the NPD patterns. Note that the Al peaks from pressure cell won't change with pressure, we normalize the peak intensities based on the Al(111) peak, and compare these NPD patterns as shown in Fig.S21. One can find that the peak intensity of $(000)^{ \pm}$magnetic satellite keeps almost unchanged at pressures $0 \mathrm{kbar}, 2 \mathrm{kbar}$, and $4 \mathrm{kbar}$, but shows a sharp increase when the pressure further increases to 6kbar, which suggests that a transition of spin-structure occurs.

The refined results were summarized in Table 1 (see main text). As representatives, Fig. S22a and Fig.S22b display the refined NPD patterns under $0 \mathrm{kbar}$ and $6 \mathrm{kbar}$ at $120 \mathrm{~K}$, respectively. To eliminate the influence from $\mathrm{Al}$ diffraction peaks, we exclude all the $\mathrm{Al}$ diffraction data during the refinement process, as shown in Fig.S22. The quality of refinements can be satisfactory. Careful refinements indicated the spiral configuration, as well as the spin moment, remains almost unchanged under $\mathrm{P} \leq 4 \mathrm{kbar}$ (Table 1). However, as the pressure reaches $6 \mathrm{kbar}$, the optimal parameters tunes to be $\psi=45^{\circ}, \theta=0^{\circ}$, and $\varphi=0^{\circ}$, corresponding to a conical spiral structure, we name it $45^{\circ}$-CoS-FM ${ }^{\text {a }}$ (Fig.3c). Meanwhile, the refined moment drops from $2.80(3) \mu_{\mathrm{B}}$ (0kbar) to $2.08(3) \mu_{\mathrm{B}}(6.0 \mathrm{kbar})$. All these are in line with the macroscopic magnetic measurements (Fig.3a), noting that the refined moments $\left(\mathrm{M}_{\mathrm{Mn}(\mathrm{Fe})}\right)$ from NPD are nearly the same as the saturation moments (Ms) from the M-H curves at corresponding conditions (Table 1, Table S3). Such good consistency also illustrates the reliability of refined results. 


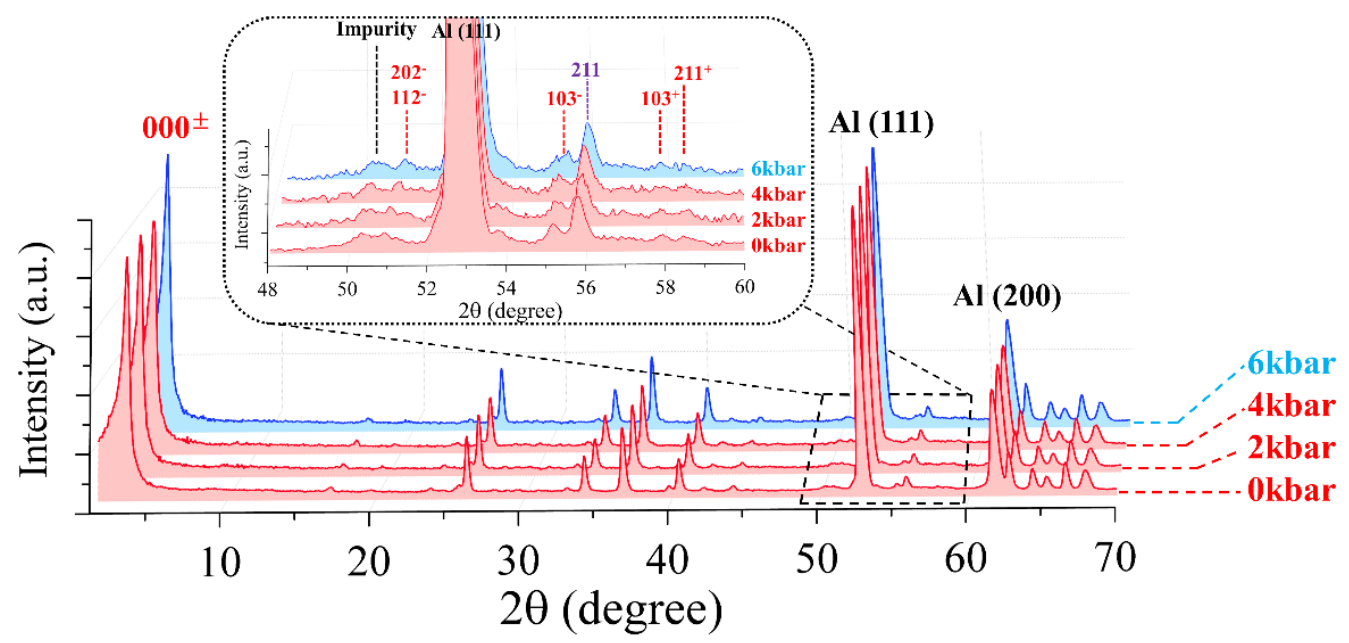

Fig.S21 Variable pressure NPD patterns at 120K, where the peak intensities are normalized based on the $\mathrm{Al}(111)$ peak (pressure cell holding sample). Inset: the NPD patterns in the $2 \theta$ range of $48^{\circ}-60^{\circ}$, where the incommensurate magnetic satellites (red), orthorhombic crystal reflections (purple), and impurity peaks possibly from device environment (black) are indexed.
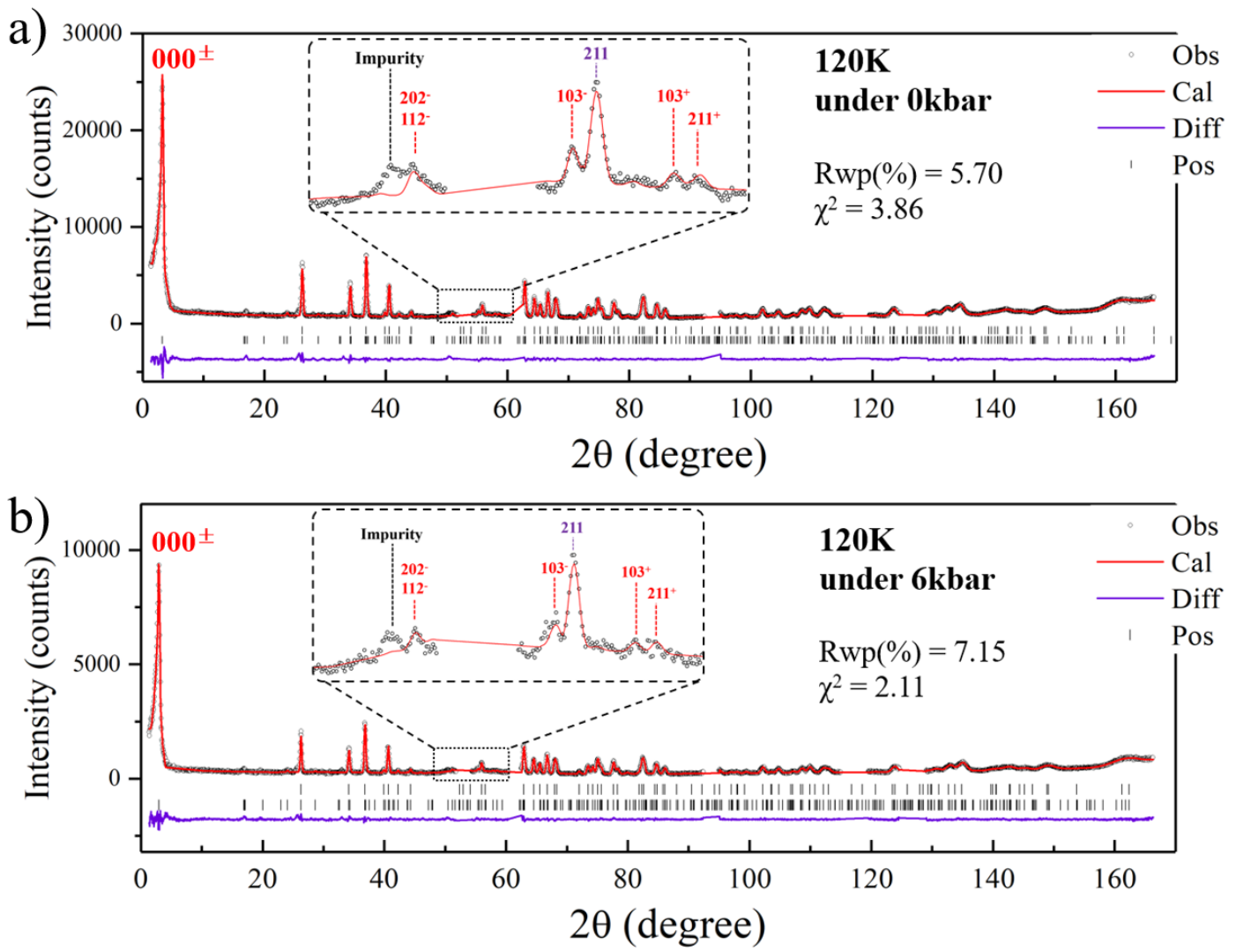

Fig.S22 Observed (black circle), calculated (red line) NPD patterns, their difference (purple line), peak positions (black bar) for $\mathrm{Mn}_{0.87} \mathrm{Fe}_{0.13} \mathrm{NiGe}$ collected at $120 \mathrm{~K}$ under a) $0 \mathrm{kbar}$ and b) $6 \mathrm{kbar}$, respectively. For clarity, the details of NPD patterns within $48^{\circ}-60^{\circ}$ are given in the corresponding inset. 
References:

[S1] Shi, K. W.; Sun, Y.; Yan, J.; Deng, S. H.; Wang, L.; Wu, H.; Hu, P. W.; Hu, H. Q.; Malik, M. I.; Huang, Q. Z.; Wang, C. Baromagnetic Effect in antiperovskite $\mathrm{Mn}_{3} \mathrm{Ga}_{0.95} \mathrm{~N}_{0.94}$ by neutron powder diffraction analysis. $A d v$. Mater. 2016, 28, 3761-3767.

[S2] Kekalo, I. B.; Nemova, O. Y.; Taranitchev, V. E. Piezomagnetic coefficients of amorphous alloys with low magnetostriction. J. Magn. Magn. Mater. 1996, 157, 181-182.

[S3] Shen, F. R.; Zhou, H. B.; Hu, F. X.; Wang, J. T.; Deng, S. H.; Wang, B. T.; Wu, H.; Huang, Q. H.; Wang, J.; Chen, J.; He, L. H.; Hao, J. Z.; Yu, Z. B.; Liang, F. X.; Liang, T. J.; Sun, J. R.; Shen, B. G. Cone-spiral magnetic ordering dominated lattice distortion and giant negative thermal expansion in Fe-doped MnNiGe compounds. Mater. Horiz. 2020, 7, 804-810.

[S4] Liu, E. K.; Wang, W. H.; Feng, L.; Zhu, W.; Li, G. J.; Chen, J. L.; Zhang, H. W.; Wu, G. H.; Jiang, C. B.; Xu, H. B.; Frank, D. B. Stable magnetostructural coupling with tunable magnetoresponsive effects in hexagonal ferromagnets. Nat. Commun. 2012, 3, 873.

[S5] Kresse, G.; Hafner, J. Ab initio molecular dynamics for liquid mentals. Phys. Rev. B 1993, 47, 558-561.

[S6] Kresse, G.; Hafner, J. Efficient iterative schemes for ab initio total-energy calculations using a plane-wave basis set. Phys. Rev. B 1996, 54, 11169-11186.

[S7] Kresse, G.; Joubert, D. From ultrasoft pseudopotentials to the projector augmented-wave method. Phys. Rev. B 1996, 59, 1758-1775.

[S8] Blöchl, P. E. Projector augmented-wave method. Phys. Rev. B 1994, 50, 17953-17979.

[S9] Predew, J. P.; Wang, Y. Accurate and simple analytic representation of the electron-gas correlation energy. Phys. Rev. B 1992, 45, 13244-13249.

[S10] Bazela, W.; Szytula, A.; Todorovic, J.; Tomkowicz, Z.; Zieba, A. Crystal and magnetic structure of NiMnGe. Phys. Status Solidi A 1976, 38, 721.

[S11] Matsukura, F.; Ohno, H.; Shen, A.; Sugawara, Y. Transport properties and origin of ferromagnetism in (Ga,Mn)As. Phys. Rev. B 1998, 57, 2037-2040.

[S12] Gercsi, Z.; Sandeman, K. G. Structurally driven metamagnetism in MnP and related Pnma compounds. Phys. Rev. B 2010, 81, 224426.

[S13] Gercsi, Z.; Hono, K.; Sandeman, K. G. Designed metamagnetism in CoMnGe ${ }_{1-\mathrm{x}} \mathrm{P}_{\mathrm{x}}$. Phys. Rev. B 2011, 83, 174403.

[S14] Bazela, W.; Szytula, A.; Todorovic J.; Zieba, A. Crystal and magnetic structure of the NiMnGel-n $\mathrm{Si}_{\mathrm{n}}$ system. Phys. Status Solidi A 1981, 64, 367-378.

[S15] Sredniawa, B.; Zach, R.; Fornal, P.; Duraj, R.; Bombik, A.; Tobola, J.; Kaprzyk, S.; Niziol, S.; Fruchart, D.; Bacmann, M.; Fruchart, R.; Stanek, J. Crystal structure, magnetic and electronic properties of $\mathrm{CoxFe}_{1-\mathrm{XMnP}}$ system. J. Alloys Compd. 2001, 317, 266-273.

[S16] Niziol, S.; Bombik, A.; Bazela, W.; Szytula, A.; Fruchart, D. Crystal and magnetic structure of CoxNi1-xMnGe system. J. Magn. Magn. Mater. 1982, 27, 281-292.

[S17] Barcza, A.; Gercsi, Z.; Knight, K. S.; Sandeman, K. G. Giant magnetoelastic coupling in a metallic helical metamagnet. Phys. Rev. Lett. 2010, 104, 247202.

[S18] Lizarraga, R.; Nordstrom, L.; Bergqvist, L.; Bergman, A.; Sjostedt, E.; Mohn, P.; Eriksson, O. Conditions for noncollinear instabilities of ferromagnetic materials. Phys. Rev. Lett. 2004, 93, 107205.

[S19] Shull, C. G.; Wollan, E. O.; Koehler, W. C. Neutron scattering and polarization by ferromagnetic materials. Phys. Rev. 1951, 84, 912-921.

[S20] Rietveld, H. M. A profile refinement method for nuclear and magnetic structure. J. Appl. Cryst. 1969, 2, 65 71. 